\title{
Distortion Bounds for Transmitting Correlated Sources with Common Part over MAC
}

\author{
Lei Yu, Houqiang Li, Senior Member, IEEE, and Chang Wen Chen, Fellow, IEEE
}

\begin{abstract}
This paper investigates the joint source-channel coding problem of sending two correlated memoryless sources with common part over a memoryless multiple access channel (MAC). An inner bound and two outer bounds on the achievable distortion region are derived. In particular, they respectively recover the existing bounds for several special cases, such as communication without common part, lossless communication, and noiseless communication. When specialized to quadratic Gaussian communication case, the inner bound and outer bound are used to generate two new bounds. Numerical result shows that common part improves the performance of such distributed communication system.
\end{abstract}

\section{INTRODUCTION}

The joint source-channel coding (JSCC) problem of transmitting correlated sources (with common part) over multiple access channel was first studied by Cover et al. [10] in which a bivariate finite-alphabet source is to be transmitted losslessly over a two-to-one multiple-access channel. As a lossy version of such JSCC problem, Minero et al. [7] considered the achievable distortion region of sending memoryless correlated source without common part over memoryless multiple access channel, and they derived an inner bound using a unified framework of hybrid coding (although the result still holds for the communication with common part, however it will become loose especially when the correlated sources are identical). This unified hybrid coding [7] generalizes the JSCC scheme given by Cover et al. [10], and can recover the achievability result given in [10]. In addition, specialized to quadratic Gaussian communication case, the inner bound in [7] can also recover the performance of hybrid coding given by Lapidoth et al. [12].

As for the converse part, Cover et al. [10] gave a tight but uncomputable (multi-letter) outer bound for lossless communication case, and Kang et al. [17] single-letterized this outer bound by utilizing a data processing inequality on maximal correlation coefficient. For lossy case, Lapidoth et al. [12] gave an outer bound for quadratic Gaussian communication utilizing a similar data processing inequality as well. Recently, Lapidoth et al. [18] also derived a new outer bound by the technology of introducing an auxiliary random variable (or remote source). However, the necessary condition of [18] is weaker than the one of [12] due to no data processing inequality applied in the single-letterization processing [18].

Lei Yu and Houqiang Li are with the Department of Electronic Engineering and Information Science, University of Science and Technology of China, Hefei, China (e-mail: yulei@ustc.edu.cn, lihq@ustc.edu.cn). Chang Wen Chen is with the Department of Computer Science and Engineering, State University of New York at Buffalo, Buffalo, USA (e-mail: chencw@buffalo.edu).
As a lossy version of the problem (with common part) studied by Cover et al. [10], in this paper, we consider JSCC of transmitting two memoryless correlated sources with common part over multiple access channel, and give an inner bound and two outer bounds on the achievable distortion region. For the inner bound, we propose an extended version of hybrid coding by adding common part into the hybrid coding [7] that is designed for the case with no common part, and hence our inner bound can recover the performance of the hybrid coding [7] by setting the common part to be empty. In addition, the outer bound is derived by introducing auxiliary random variables (or remote sources) as in [1]-[6], and it can recover the existing outer bounds when common part is absent at both encoders. When specialized to Gaussian communication with Gaussian common part, our bounds reduce to a new inner bound and a new outer bound.

The rest of this paper is organized as follows. Section II summarizes basic notations, and formulates the problem. Section III gives the main results for transmitting memoryless sources over memoryless MAC problem. Section IV gives the main results for Gaussian communication case. Finally, Section $\mathrm{V}$ gives the concluding remarks.

\section{Problem Formulation AND PRELIMINARIES}

Consider the correlated sources $S_{1}$ and $S_{2}$ have common part in sense of Gács-Körner-Witsenhausen common information [13], [14].

Definition 1. $S_{0}$ is a common part of two correlated sources $S_{1}$ and $S_{2}$ if there exist two functions $f_{k}: \mathcal{S}_{k} \mapsto \mathcal{S}_{0}, k=1,2$ such that $S_{0}=f_{1}\left(S_{1}\right)=f_{2}\left(S_{2}\right)$ with probability one, where $\mathcal{S}_{k}$ denotes the alphabet of $S_{k}, k=0,1,2$. We say that $S_{1}$ and $S_{2}$ have a common part if there exists a such $S_{0}$ as a common part of $S_{1}$ and $S_{2}$.

Now consider the problem transmitting correlated sources over a multiple access channel as shown in Fig. 1. The sender $k=1,2$ first codes discrete memoryless source $S_{k}^{n}$ into $X_{k}^{n}$ using a source-channel code, then transmits $X_{k}^{n}$ to a common receiver through a discrete memoryless multiple access channel (DM-MAC) $p_{Y \mid X_{1}, X_{2}}$, and finally, the receiver produces source reconstructions $\hat{S}_{1}^{n}$ and $\hat{S}_{2}^{n}$ from the received signal $Y^{n}$.

Definition 2. An $n$-length source-channel code is defined by the two encoding functions $x_{k}^{n}: \mathcal{S}_{k}^{n} \mapsto \mathcal{X}_{k}^{n}, k=1,2$ and two decoding functions $\hat{s}_{k}: \mathcal{Y}^{n} \mapsto \mathcal{S}_{k}^{n}, k=1,2$, where $\hat{\mathcal{S}}_{k}, \mathcal{X}_{k}$ and $\mathcal{Y}$ are the alphabet of source reconstruction $\hat{S}_{k}$, channel input $X_{k}$, and channel output $Y$. 


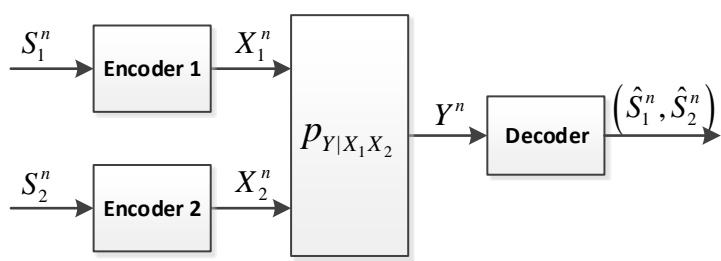

Fig. 1. Communication of memoryless correlated sources over a DM-MAC.

For any $n$-length source-channel code, the induced distortion is defined as

$$
\mathbb{E} d_{k}\left(S_{k}^{n}, \hat{S}_{k}^{n}\right)=\frac{1}{n} \sum_{t=1}^{n} \mathbb{E} d_{k}\left(S_{k, t}, \hat{S}_{k, t}\right)
$$

for $k=1,2$, where $d_{k}\left(s_{k}, \hat{s}_{k}\right): \mathcal{S}_{k} \times \hat{\mathcal{S}}_{k} \mapsto[0,+\infty]$ is a distortion measure function for source $S_{k}$.

Definition 3. For transmitting sources $\left(S_{1}, S_{2}\right)$ over MAC $p_{Y \mid X_{1}, X_{2}}$, we say the distortion tuple $\left(D_{1}, D_{2}\right)$ is achievable, if there exists a sequence of source-channel codes such that

$$
\limsup _{n \rightarrow \infty} \mathbb{E} d_{k}\left(S_{k}^{n}, \hat{S}_{k}^{n}\right) \leq D_{k} .
$$

Definition 4. For transmitting source $\left(S_{1}, S_{2}\right)$ over MAC $p_{Y \mid X_{1}, X_{2}}$, the admissible distortion region is defined as

$$
\mathcal{R} \triangleq\left\{\left(D_{1}, D_{2}\right):\left(D_{1}, D_{2}\right) \text { is achievable }\right\} .
$$

In symmetric case,

$$
\mathcal{R}_{\text {sym }} \triangleq\{D:(D, D) \text { is achievable }\} .
$$

\section{General Communication}

Now, we bound the distortion region for correlated sources communication over MAC. We first define a distortion region

$\mathcal{R}^{(i)}=\left\{\left(D_{1}, D_{2}\right):\right.$ There exist some pmf $p_{V_{0} \mid S_{0}} p_{V_{1} \mid S_{1}, V_{0}} p_{V_{2} \mid S_{2}, V_{0}}$, and functions $x_{k}\left(v_{0}, v_{k}, s_{k}\right), \hat{s}_{k}\left(v_{0}, v_{1}, v_{2}, y\right), k=1,2$ such that

$\mathbb{E} d_{k}\left(S_{k}, \hat{S}_{k}\right) \leq D_{k}, k=1,2$,

$I\left(V_{1} ; S_{1} \mid V_{0} V_{2}\right)<I\left(V_{1} ; Y \mid V_{0} V_{2}\right)$,

$I\left(V_{2} ; S_{2} \mid V_{0} V_{1}\right)<I\left(V_{2} ; Y \mid V_{0} V_{1}\right)$,

$I\left(V_{1} V_{2} ; S_{1} S_{2} \mid V_{0}\right)<I\left(V_{1} V_{2} ; Y \mid V_{0}\right)$,

$\left.I\left(V_{0} V_{1} V_{2} ; S_{1} S_{2}\right)<I\left(V_{0} V_{1} V_{2} ; Y\right)\right\}$.

and another two distortion regions ${ }^{1}$

$\mathcal{R}_{1}^{(o)}=$

$\left\{\left(D_{1}, D_{2}\right)\right.$ : For any $p_{U_{[1: L]} \mid S_{1}, S_{2}}$, there exist some pmf $p_{\hat{S}_{1}, \hat{S}_{2} \mid S_{1}, S_{2}, U}$ and $p_{Q} \prod p_{S_{1}, S_{2}}\left(s_{1, i}, s_{2, i}\right) p_{U_{[1: L]} \mid S_{1}, S_{2}}\left(u_{[1: L], i} \mid s_{1, i}, s_{2, i}\right) p_{X_{1} \mid S_{1}^{n}, Q} p_{X_{2} \mid S_{2}^{n}, Q}$ such that $\mathbb{E} d_{k}\left(S_{k}, \hat{S}_{k}\right) \leq D_{k}, k=1,2$,

$I\left(S_{1} S_{2} ; \hat{S}_{1} \hat{S}_{2} \mid U_{\mathcal{A}}\right) \leq I\left(X_{1} X_{2} ; Y \mid U_{\mathcal{A}}^{n} Q\right)$ for any $\left.\mathcal{A} \subseteq[1: L]\right\}$.

${ }^{1}$ The $L$ in $\mathcal{R}_{1}^{(o)}$ is an arbitrary positive integer.

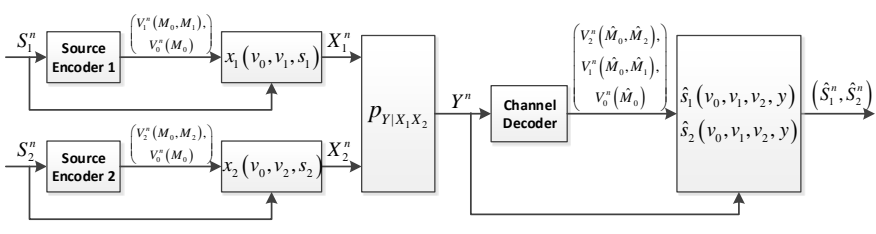

Fig. 2. The hybrid coding used to prove the inner bound in Theorem 1.

and

$\mathcal{R}_{2}^{(o)}=$

$\left\{\left(D_{1}, D_{2}\right)\right.$ : For any $p_{U \mid S_{1}, S_{2}}$ such that $S_{1} \rightarrow\left(S_{0}, U\right) \rightarrow S_{2}$,

there exist some pmf $p_{\hat{S}_{1}, \hat{S}_{2} \mid S_{1}, S_{2}, U}$ and

$p_{Q} \prod p_{S_{1}, S_{2}}\left(s_{1, i}, s_{2, i}\right) p_{U \mid S_{1}, S_{2}}\left(u_{i} \mid s_{1, i}, s_{2, i}\right) p_{X_{1} \mid S_{1}^{n}, Q} p_{X_{2} \mid S_{2}^{n}, Q}$

such that $\mathbb{E} d_{k}\left(S_{k}, \hat{S}_{k}\right) \leq D_{k}, k=1,2$,

$I\left(S_{1} S_{2} ; \hat{S}_{1} \hat{S}_{2}\right) \leq I\left(X_{1} X_{2} ; Y \mid Q\right)$,

$I\left(S_{1} S_{2} ; \hat{S}_{1} \hat{S}_{2} \mid S_{0}\right) \leq I\left(X_{1} X_{2} ; Y \mid S_{0}^{n} Q\right)$,

$I\left(S_{1} ; \hat{S}_{1} \hat{S}_{2} \mid S_{2}\right) \leq I\left(X_{1} ; Y \mid X_{2} S_{2}^{n} Q\right)$,

$I\left(S_{2} ; \hat{S}_{1} \hat{S}_{2} \mid S_{1}\right) \leq I\left(X_{2} ; Y \mid X_{1} S_{1}^{n} Q\right)$,

$I\left(S_{1} S_{2} ; \hat{S}_{1} \hat{S}_{2} \mid S_{0} U\right) \leq I\left(X_{1} X_{2} ; Y \mid S_{0}^{n} U^{n} Q\right)$,

$I\left(S_{1} ; \hat{S}_{1} \hat{S}_{2} \mid S_{2} U\right) \leq I\left(X_{1} ; Y \mid X_{2} S_{2}^{n} U^{n} Q\right)$,

$\left.I\left(S_{2} ; \hat{S}_{1} \hat{S}_{2} \mid S_{1} U\right) \leq I\left(X_{2} ; Y \mid X_{1} S_{1}^{n} U^{n} Q\right)\right\}$.

Note that in $\mathcal{R}_{2}^{(o)}, p_{U \mid S_{1}, S_{2}}$ such that $S_{1} \rightarrow\left(S_{0}, U\right) \rightarrow S_{2}$ always exists, since $S_{1} \rightarrow\left(S_{0}, S_{1}\right) \rightarrow S_{2}$ and $S_{1} \rightarrow\left(S_{0}, S_{2}\right) \rightarrow$ $S_{2}$. In addition, it is easy to verify that for such $p_{U \mid S_{1}, S_{2}}$, the random variables $\left(S_{1}^{n}, S_{2}^{n}, S_{0}^{n}, U^{n}, X_{1}, X_{2}, Q\right)$ in $\mathcal{R}_{2}^{(o)}$ satisfy $X_{1} \rightarrow\left(S_{1}^{n}, Q\right) \rightarrow\left(S_{0}^{n}, U^{n}, Q\right) \rightarrow\left(S_{2}^{n}, Q\right) \rightarrow X_{2}$. Now we give the following theorem.

Theorem 1. For transmitting sources $\left(S_{1}, S_{2}\right)$ with common part $S_{0}$ over MAC $p_{Y \mid X_{1}, X_{2}}$,

$$
\mathcal{R}^{(i)} \subseteq \mathcal{R} \subseteq \mathcal{R}_{1}^{(o)} \subseteq \mathcal{R}_{2}^{(o)} .
$$

Remark 1. The inner bound in Theorem 1 can be easily extended to Gaussian or any other well-behaved continuousalphabet source-channel pair by standard discretization method [9, Thm. 3.3], and moreover for this case the outer bound still holds. Theorem 1 can be also extended to the case of source-channel bandwidth mismatch, where $m$ samples of memoryless correlated sources are transmitted through $n$ uses of a DM-MAC. This can be accomplished by replacing the source and channel symbols in Theorem 1 by supersymbols of lengths $m$ and $n$, respectively. Besides, Theorem 1 can be also extended to the problem with channel input cost (by adding channel input constraint).

Proof: The proof of $\mathcal{R}^{(i)} \subseteq \mathcal{R} \subseteq \mathcal{R}_{1}^{(o)}$ is given in Appendix A. Now we show that $\mathcal{R}_{1}^{(o)} \subseteq \mathcal{R}_{2}^{(o)}$. Actually $\mathcal{R}_{2}^{(o)}$ is a straightforward consequence of $\mathcal{R}_{1}^{(o)}$. Choose $L=4, U_{1}=S_{1}, U_{2}=S_{2}, U_{3}=S_{0}, U_{4}=U$ in $\mathcal{R}_{1}^{(o)}$, where $U \mid S_{1}, S_{2}$ follows $p_{U \mid S_{1}, S_{2}}$. Then setting $\mathcal{A}=$ $\emptyset,\{1\},\{2\},\{3\},\{1,4\},\{2,4\},\{3,4\}$ in $\mathcal{R}_{1}^{(o)}$ respectively gives the inequalities in $\mathcal{R}_{2}^{(o)}$. Hence $\mathcal{R}_{1}^{(o)} \subseteq \mathcal{R}_{2}^{(o)}$. 
The inner bound $\mathcal{R}^{(i)}$ in Theorem 1 is achieved by a unified hybrid coding scheme depicted in Fig. 2. In this scheme, the codebook has a layered (or superposition) structure, and consists of randomly and independently generated codewords $\left(V_{0}^{n}\left(m_{0}\right), V_{1}^{n}\left(m_{0}, m_{1}\right), V_{2}^{n}\left(m_{0}, m_{2}\right)\right)$, $\left(m_{0}, m_{1}, m_{2}\right) \in \prod_{i=0}^{2}\left[1: 2^{n r_{i}}\right]$, where $\left(r_{0}, r_{1}, r_{2}\right)$ denote the rates of the digital information part and their values are given in Appendix A. At encoder sides, upon source sequence $S_{k}^{n}, k=1,2$, the encoder $k$ first generates the common source $S_{0}^{n}$, and produces a common digital message $M_{0}$ from $S_{0}^{n}$ by joint typicality encoding. Then upon $M_{0}$ and $S_{k}^{n}$, the encoder $k$ produces a private digital messages $M_{k}$. Finally, the codeword $\left(V_{0}^{n}\left(M_{0}\right), V_{k}^{n}\left(M_{0}, M_{k}\right)\right)$ and the source sequence $S_{k}^{n}$ are used to generate channel input $X_{k}^{n}$ by symbol-bysymbol mapping $x_{k}\left(v_{0}, v_{k}, s_{k}\right)$. At decoder side, upon received signal $Y^{n}$, the decoder reconstruct $\left(M_{0}, M_{1}, M_{2}\right)$ (and also $\left.\left(V_{0}^{n}\left(m_{0}\right), V_{1}^{n}\left(m_{0}, m_{1}\right), V_{2}^{n}\left(m_{0}, m_{2}\right)\right)\right)$ losslessly by joint typicality decoding, and then it produces $\hat{S}_{k}^{n}, k=1,2$ by symbol-by-symbol mapping $\hat{s}_{k}\left(v_{0}, v_{1}, v_{2}, y\right)$. Such a scheme could achieve any $\left(D_{1}, D_{2}\right)$ in the inner bound $\mathcal{R}^{(i)}$.

Note that our hybrid coding is a extension of the hybrid coding in [7] to the case of correlated sources with common part. By setting the common part as empty, i.e., $V_{0}=\emptyset$, our result can recover the performance of the hybrid coding in [7]; see the special cases in the following.

The outer bounds $\mathcal{R}_{1}^{(o)}$ and $\mathcal{R}_{2}^{(o)}$ in Theorem 1 are derived by introducing auxiliary random variables (or remote sources) $U_{[1: L]}^{n}$ or $U^{n}$. This bounding technology was originated in the multiple description problem by Ozarow [1], and sequentially used in the distributed source coding problem by Wagner $e t$ al. [2] and the source broadcast problem [3], [4], [5], [6]. As these works, in our proof one or multiple additional random variables beyond those in the original problem are introduced. Besides, for $\mathcal{R}_{2}^{(o)}$ the auxiliary random variable $U$ is restricted to following the Markov chain $S_{1} \rightarrow\left(S_{0}, U\right) \rightarrow S_{2}$. This is inspired the work of Wagner et al. [2], where they showed that introducing an auxiliary random variable that follows a Markov chain structure is sufficient to achieve the tight bound for distributed source coding problem. Observe that our problem is a extension of distributed source coding problem to the noisy communication case, hence such a Markov chain structure is also used in our bound. Besides, such a Markov chain structure also makes a sequence of data processing inequalities available, which in turn generates some simpler bounds (see Section IV-C).

\section{A. Special Cases}

- Lossy Communication without Common Part

When there is no common part, the inner bound in Theorem 1 reduces to [7, Thm. 1], i.e.,

$\mathcal{R}^{(i)}=\left\{\left(D_{1}, D_{2}\right)\right.$ : There exist some pmf $p_{V_{0}} p_{V_{1} \mid S_{1}, V_{0}} p_{V_{2} \mid S_{2}, V_{0}}$, and functions $x_{k}\left(v_{0}, v_{k}, s_{k}\right), \hat{s}_{k}\left(v_{0}, v_{1}, v_{2}, y\right), k=1,2$ such that $\mathbb{E} d_{k}\left(S_{k}, \hat{S}_{k}\right) \leq D_{k}, k=1,2$, $I\left(V_{1} ; S_{1} \mid V_{2} V_{0}\right)<I\left(V_{1} ; Y \mid V_{2} V_{0}\right)$, $I\left(V_{2} ; S_{2} \mid V_{1} V_{0}\right)<I\left(V_{2} ; Y \mid V_{1} V_{0}\right)$, $\left.I\left(V_{1} V_{2} ; S_{1} S_{2} \mid V_{0}\right)<I\left(V_{1} V_{2} ; Y \mid V_{0}\right)\right\}$.
In this case, $V_{0}$ is independent of $S_{1}$ and $S_{2}$, and it becomes a timesharing auxiliary random variable. In addition, the outer bound $\mathcal{R}_{2}^{(o)}$ reduces to a new outer bound

$\mathcal{R}^{(o)}=\left\{\left(D_{1}, D_{2}\right):\right.$ For any $p_{U \mid S_{1}, S_{2}}$ such that $S_{1} \rightarrow U \rightarrow S_{2}$,

there exist some pmf $p_{\hat{S}_{1}, \hat{S}_{2} \mid S_{1}, S_{2}, U}$ and

$p_{Q} \prod p_{S_{1}, S_{2}}\left(s_{1, i}, s_{2, i}\right) p_{U \mid S_{1}, S_{2}}\left(u_{i} \mid s_{1, i}, s_{2, i}\right) p_{X_{1} \mid S_{1}^{n}, Q} p_{X_{2} \mid S_{2}^{n}, Q}$

such that $\mathbb{E} d_{k}\left(S_{k}, \hat{S}_{k}\right) \leq D_{k}, k=1,2$,

$I\left(S_{1} S_{2} ; \hat{S}_{1} \hat{S}_{2}\right) \leq I\left(X_{1} X_{2} ; Y \mid Q\right)$,

$I\left(S_{1} ; \hat{S}_{1} \hat{S}_{2} \mid S_{2}\right) \leq I\left(X_{1} ; Y \mid X_{2} S_{2}^{n} Q\right)$,

$I\left(S_{2} ; \hat{S}_{1} \hat{S}_{2} \mid S_{1}\right) \leq I\left(X_{2} ; Y \mid X_{1} S_{1}^{n} Q\right)$,

$I\left(S_{1} S_{2} ; \hat{S}_{1} \hat{S}_{2} \mid U\right) \leq I\left(X_{1} X_{2} ; Y \mid U^{n} Q\right)$,

$I\left(S_{1} ; \hat{S}_{1} \hat{S}_{2} \mid S_{2} U\right) \leq I\left(X_{1} ; Y \mid X_{2} S_{2}^{n} U^{n} Q\right)$,

$\left.I\left(S_{2} ; \hat{S}_{1} \hat{S}_{2} \mid S_{1} U\right) \leq I\left(X_{2} ; Y \mid X_{1} S_{1}^{n} U^{n} Q\right)\right\}$.

- Lossless Communication with Common Part

When specialized to lossless communication of correlated source with common part, by setting $V_{0}=\left(S_{0}, W\right), V_{k}=$ $\left(S_{k}, X_{k}\right), x_{k}\left(v_{0}, v_{k}, s_{k}\right)=x_{k}, \hat{s}_{k}\left(v_{0}, v_{1}, v_{2}, y\right)=s_{k}, k=$ 1,2 where $W$ is a random variable independent of $S_{1}$ and $S_{2}$, the inner bound in Theorem 1 recovers the inner bound [10, Thm. 1] on the admissible sources region, i.e.,

$\mathcal{R}^{(i)}=\left\{p_{S_{1}, S_{2}}\right.$ : There exist some pmf $p_{W} p_{X_{1} \mid S_{1}, W} p_{X_{2} \mid S_{2}, W}$ such that

$H\left(S_{1} \mid S_{2}\right) \leq I\left(X_{1} ; Y \mid X_{2} S_{2} W\right)$,

$H\left(S_{2} \mid S_{1}\right) \leq I\left(X_{2} ; Y \mid X_{1} S_{1} W\right)$,

$H\left(S_{1} S_{2} \mid S_{0}\right) \leq I\left(X_{1} X_{2} ; Y \mid S_{0} W\right)$,

$\left.H\left(S_{1} S_{2}\right) \leq I\left(X_{1} X_{2} ; Y\right)\right\}$.

In addition, the outer bound in Theorem 1 reduces to a new outer bound

$\mathcal{R}^{(o)}=\left\{p_{S_{1}, S_{2}}:\right.$ For any $p_{U \mid S_{1}, S_{2}}$ such that $S_{1} \rightarrow\left(S_{0}, U\right) \rightarrow S_{2}$,

there exist some pmf

$p_{Q} \prod p_{S_{1}, S_{2}}\left(s_{1, i}, s_{2, i}\right) p_{U \mid S_{1}, S_{2}}\left(u_{i} \mid s_{1, i}, s_{2, i}\right) p_{X_{1} \mid S_{1}^{n}, Q} p_{X_{2} \mid S_{2}^{n}, Q}$ such that

$H\left(S_{1} S_{2}\right) \leq I\left(X_{1} X_{2} ; Y \mid Q\right)$,

$H\left(S_{1} \mid S_{2}\right) \leq I\left(X_{1} ; Y \mid X_{2} S_{2}^{n} Q\right)$,

$H\left(S_{2} \mid S_{1}\right) \leq I\left(X_{2} ; Y \mid X_{1} S_{1}^{n} Q\right)$,

$H\left(S_{1} S_{2} \mid S_{0}\right) \leq I\left(X_{1} X_{2} ; Y \mid S_{0}^{n} Q\right)$,

$H\left(S_{1} S_{2} \mid S_{0} U\right) \leq I\left(X_{1} X_{2} ; Y \mid S_{0}^{n} U^{n} Q\right)$,

$H\left(S_{1} \mid S_{2} U\right) \leq I\left(X_{1} ; Y \mid X_{2} S_{2}^{n} U^{n} Q\right)$,

$\left.H\left(S_{2} \mid S_{1} U\right) \leq I\left(X_{2} ; Y \mid X_{1} S_{1}^{n} U^{n} Q\right)\right\}$.

Furthermore, if $\left(S_{1}, S_{2}\right)$ satisfy $S_{1} \rightarrow S_{0} \rightarrow S_{2}$, then

$$
\begin{aligned}
\mathcal{R}=\mathcal{R}^{(i)}=\mathcal{R}^{(o)}= & \left\{p_{S_{1}, S_{2}}:\right. \text { There exist some pmf } \\
& p_{W} p_{X_{1} \mid S_{1}, W} p_{X_{2} \mid S_{2}, W} \text { such that } \\
& H\left(S_{1} \mid S_{2}\right) \leq I\left(X_{1} ; Y \mid X_{2} S_{2} W\right), \\
& H\left(S_{2} \mid S_{1}\right) \leq I\left(X_{2} ; Y \mid X_{1} S_{1} W\right), \\
& H\left(S_{1} S_{2} \mid S_{0}\right) \leq I\left(X_{1} X_{2} ; Y \mid S_{0} W\right), \\
& \left.H\left(S_{1} S_{2}\right) \leq I\left(X_{1} X_{2} ; Y\right)\right\} .
\end{aligned}
$$

This implies the admissible sources region for transmitting the correlated sources that are conditionally independent given the common part has been characterized completely. As a counterpart, the admissible sources region for broadcasting conditionally independent sources has been given in [6]. 
When $S_{0}, S_{1}, S_{2}$ are independent, the distortion region can be used to derive the capacity region of Multiple Access Channel with Common Message.

- Multiple Access Channel with Common Message Consider lossless communication of independent sources $S_{0}, S_{1}, S_{2}$ with $H\left(S_{k}\right)=R_{k}, k=0,1,2$, then the problem becomes Multiple Access Channel with Common Message [11]. Specialized to this case, (13) reduces to the capacity region [11, Eqn. 11], i.e.,

$$
\begin{aligned}
& \mathcal{R}=\mathcal{R}^{(i)}=\mathcal{R}^{(o)}=\left\{\left(R_{0}, R_{1}, R_{2}\right):\right. \text { There exist some pmf } \\
& p_{W} p_{X_{1} \mid W} p_{X_{2} \mid W} \text { such that } \\
& R_{1} \leq I\left(X_{1} ; Y \mid X_{2} W\right) \\
& R_{2} \leq I\left(X_{2} ; Y \mid X_{1} W\right) \\
& R_{1}+R_{2} \leq I\left(X_{1} X_{2} ; Y \mid W\right) \\
&\left.R_{0}+R_{1}+R_{2} \leq I\left(X_{1} X_{2} ; Y\right)\right\} .
\end{aligned}
$$

- Distributed Source Coding with Common Part Consider the MAC $p_{Y \mid X_{1}, X_{2}}$ is noiseless, i.e., $Y=\left(X_{1}, X_{2}\right)$, and constrain $H\left(X_{k}\right) \stackrel{\leq}{\leq} R_{k}, k=1,2$, then the problem becomes Distributed Source Coding with Common Part [8]. Set $V_{k}=\left(V_{k}, X_{k}\right), x_{k}\left(v_{0}, v_{k}, s_{k}\right)=x_{k}, \hat{s}_{k}\left(v_{0}, v_{1}, v_{2}, y\right)=$ $\hat{s}_{k}\left(v_{0}, v_{1}, v_{2}\right), k=1,2$, where $X_{1}$ and $X_{2}$ are two random variables independent of each other and other variables. Then the inner bound of Theorem 1 recovers the inner bound $[8$, Thm.1] on the achievable distortion region, i.e.,

$\mathcal{R}^{(i)}=\left\{\left(D_{1}, D_{2}\right):\right.$ There exist some pmf $p_{V_{0} \mid S_{0}} p_{V_{1} \mid S_{1}, V_{0}} p_{V_{2} \mid S_{2}, V_{0}}$, and functions $\hat{s}_{k}\left(v_{0}, v_{1}, v_{2}\right), k=1,2$ such that

$$
\begin{aligned}
& \mathbb{E} d_{k}\left(S_{k}, \hat{S}_{k}\right) \leq D_{k}, k=1,2, \\
& I\left(V_{1} ; S_{1} \mid V_{0} V_{2}\right)<R_{1}, \\
& I\left(V_{2} ; S_{2} \mid V_{0} V_{1}\right)<R_{2}, \\
& \left.I\left(V_{0} V_{1} V_{2} ; S_{1} S_{2}\right)<R_{1}+R_{2}\right\} .
\end{aligned}
$$

\section{QUADRATIC GAUSSIAN COMMUNICATION}

In this section, we apply the result for general communication to the quadratic Gaussian communication case. Consider sending jointly Gaussian sources $S_{k}=\left(S_{0}, S_{k}^{\prime}\right), k=1,2$ with $\left(S_{0}, S_{1}^{\prime}, S_{2}^{\prime}\right) \sim \mathcal{N}\left(\mathbf{0}, \Sigma_{\left(S_{0}, S_{1}^{\prime}, S_{2}^{\prime}\right)}\right)$ and $^{2}$

$$
\Sigma_{\left(S_{0}, S_{1}^{\prime}, S_{2}^{\prime}\right)}=\left(\begin{array}{ccc}
1 & \rho_{01} & \rho_{02} \\
\rho_{01} & 1 & \rho_{12} \\
\rho_{02} & \rho_{12} & 1
\end{array}\right)
$$

over a power-constrained Gaussian MAC $Y=X_{1}+X_{2}+Z$ with $\mathbb{E}\left(X_{k}^{2}\right) \leq P_{k}, k=1,2$ and $Z \sim \mathcal{N}(0,1)^{3}$. We also assume distortion is measured by quadratic distortion function on $S_{k}^{\prime}, k=1,2$, i.e., $d_{k}\left(s_{k}, \hat{s}_{k}\right)=d\left(s_{k}^{\prime}, \hat{s}_{k}\right) \triangleq$ $\left(s_{k}^{\prime}-\hat{s}_{k}\right)^{2}, k=1,2$, and source bandwidth and channel bandwidth are matched.

Without loss of generality, $\left(S_{0}, S_{1}^{\prime}, S_{2}^{\prime}\right)$ can be expressed as

$$
\begin{aligned}
& S_{1}^{\prime}=\rho_{01} S_{0}+\sqrt{1-\rho_{01}^{2}} U_{1}, \\
& S_{2}^{\prime}=\rho_{02} S_{0}+\sqrt{1-\rho_{02}^{2}} U_{2},
\end{aligned}
$$

\footnotetext{
${ }^{2}$ Throughout this paper, we use $\Sigma_{(X, Y)}$ to denote the covariance of $(X, Y)$ and $\Sigma_{X, Y}$ to denote the cross-covariance of $X$ and $Y$.

${ }^{3}$ For simplicity, we assume source variances are unit and so is the channel noise power, which can cover general cases by scaling $P_{k}$ and $D_{k}$.
}

with

$$
\begin{aligned}
& U_{1}=\beta_{1} U+\sqrt{1-\beta_{1}^{2}} B_{1}, \\
& U_{2}=\beta_{2} U+\sqrt{1-\beta_{2}^{2}} B_{2} .
\end{aligned}
$$

where $U \sim \mathcal{N}(0,1)$ and $B_{k} \sim \mathcal{N}(0,1), k=1,2$ are mutually independent Gaussian variables and also independent of $S_{0}$, and

$$
\beta_{1} \beta_{2}=\frac{\rho_{12}-\rho_{01} \rho_{02}}{\sqrt{\left(1-\rho_{01}^{2}\right)\left(1-\rho_{02}^{2}\right)}} .
$$

Obviously $S_{1} \rightarrow\left(S_{0}, U\right) \rightarrow S_{2}$ holds.

\section{A. Hybrid Coding Scheme}

In the following, we obtain the performance of hybrid coding scheme by specializing the inner bound of Theorem 1. Let

$$
\begin{aligned}
& V_{0}=S_{0}+W_{0} \\
& V_{k}=F_{k}\left(S_{0}, S_{k}, V_{0}\right)^{T}+W_{k}, k=1,2,
\end{aligned}
$$

and set $x_{k}\left(v_{0}, v_{k}, s_{k}\right), k=1,2$ to the linear functions

$$
X_{k}=G_{k}\left(S_{0}, S_{k}, V_{0}, V_{k}\right)^{T}, k=1,2,
$$

where $W_{k} \sim \mathcal{N}\left(0, \omega_{k}\right), k=0,1,2$ are mutually independent and also independent of $S_{k}, k=0,1,2$, and $F_{k}=$ $\left(f_{k, 1}, f_{k, 2}, f_{k, 3}\right)$ and $G_{k}=\left(g_{k, 1}, g_{k, 2}, g_{k, 3}, g_{k, 4}\right)$ are two row vectors of coefficients.

This induces the relationship

$\left(S_{0}, S_{1}, S_{2}, V_{0}, V_{1}, V_{2}, Y\right)^{T}=A\left(S_{0}, S_{1}, S_{2}, W_{0}, W_{1}, W_{2}, Z\right)^{T}$, where $A$ is given in (25) with

$a_{71}=g_{1,1}+g_{2,1}+g_{1,3}+g_{2,3}+g_{1,4}\left(f_{1,1}+f_{1,3}\right)+g_{2,4}\left(f_{2,1}+f_{2,3}\right)$.

Hence the covariance of $\left(S_{0}, S_{1}, S_{2}, V_{0}, V_{1}, V_{2}, Y\right)$ is given by

$$
\Sigma_{\left(S_{0}, S_{1}, S_{2}, V_{0}, V_{1}, V_{2}, Y\right)}=A \Sigma_{\left(S_{0}, S_{1}, S_{2}, W_{0}, W_{1}, W_{2}, Z\right)} A^{T} .
$$

Set $\hat{s}_{k}\left(v_{0}, v_{1}, v_{2}, y\right), k=1,2$ to the linear functions

$$
\hat{S}_{k}=\Sigma_{S_{k},\left(V_{0}, V_{1}, V_{2}, Y\right)} \Sigma_{\left(V_{0}, V_{1}, V_{2}, Y\right)}^{-1}\left(V_{0}, V_{1}, V_{2}, Y\right)^{T},
$$

then the covariance of error $E_{k} \triangleq S_{k}-\hat{S}_{k}, k=1,2$ is given by

$$
\Sigma_{E_{k}}=\Sigma_{S_{k}}-\Sigma_{S_{k},\left(V_{0}, V_{1}, V_{2}, Y\right)} \Sigma_{\left(V_{0}, V_{1}, V_{2}, Y\right)}^{-1} \Sigma_{S_{k},\left(V_{0}, V_{1}, V_{2}, Y\right)}^{T} .
$$

In addition, owing to power constraint,

$$
\Sigma_{X_{k}} \leq P_{k}
$$

where

$$
\Sigma_{X_{k}}=G_{k} \Sigma_{\left(S_{0}, S_{k}, V_{0}, V_{k}\right)} G_{k}^{T} .
$$

Substitute these random variables and functions into $\mathcal{R}^{(i)}$ in Theorem 1, then we get the performance of the hybrid coding. 


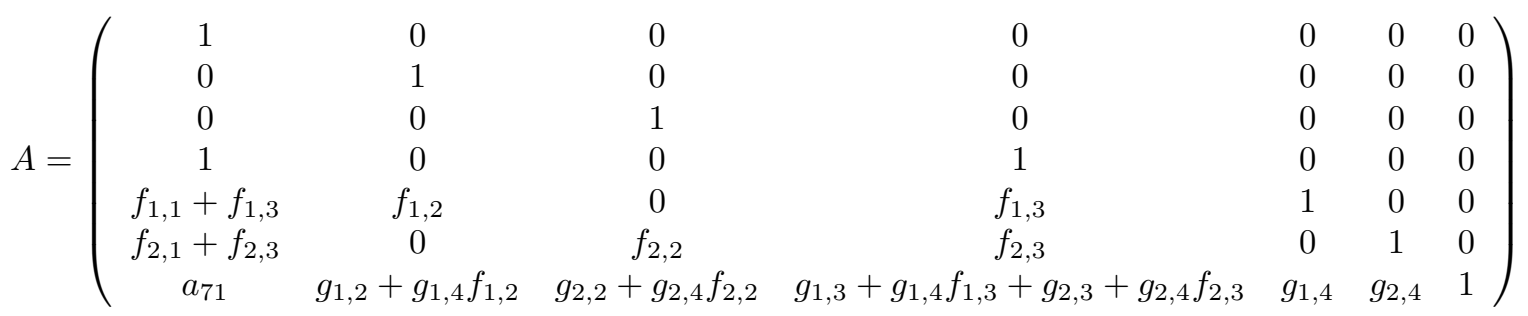

Theorem 2. For transmitting Gaussian source with common part over Gaussian MAC,

$$
\begin{aligned}
\mathcal{R} \supseteq \mathcal{R}_{h}^{(i) \triangleq} & \left\{\left(D_{1}, D_{2}\right): \text { There exist } F_{k}, G_{k}, \omega_{0}, \omega_{1}, \omega_{2}, k=1,2\right. \\
& \text { such that } \Sigma_{E_{k}} \leq D_{k}, \Sigma_{X_{k}} \leq P_{k}, k=1,2, \\
& \frac{\left|\Sigma_{\left(V_{0}, V_{2}, S_{0}, S_{1}\right)}\right|}{\left|\Sigma_{\left(V_{0}, V_{2}, V_{1}, S_{0}, S_{1}\right)}\right|}<\frac{\left|\Sigma_{\left(V_{0}, V_{2}, Y\right)}\right|}{\left|\Sigma_{\left(V_{0}, V_{2}, V_{1}, Y\right)}\right|}, \\
& \frac{\left|\Sigma_{\left(V_{0}, V_{1}, S_{0}, S_{2}\right)}\right|}{\left|\Sigma_{\left(V_{0}, V_{2}, V_{1}, S_{0}, S_{2}\right)}\right|}<\frac{\left|\Sigma_{\left(V_{0}, V_{1}, Y\right)}\right|}{\left|\Sigma_{\left(V_{0}, V_{2}, V_{1}, Y\right)}\right|}, \\
& \frac{\left|\Sigma_{\left(V_{0}, S_{0}, S_{1}, S_{2}\right)}\right|}{\left|\Sigma_{\left(V_{0}, V_{2}, V_{1}, S_{0}, S_{1}, S_{2}\right)}\right|}<\frac{\left|\Sigma_{\left(V_{0}, Y\right)}\right|}{\left|\Sigma_{\left(V_{0}, V_{2}, V_{1}, Y\right)}\right|}, \\
& \left.\frac{\left|\Sigma_{\left(S_{0}, S_{1}, S_{2}\right)}\right|}{\left|\Sigma_{\left(V_{0}, V_{2}, V_{1}, S_{0}, S_{1}, S_{2}\right)}\right|}<\frac{\left|\Sigma_{Y}\right|}{\left|\Sigma_{\left(V_{0}, V_{2}, V_{1}, Y\right)}\right|}\right\}
\end{aligned}
$$

Proof: Substitute the random variables and functions set above into $\mathcal{R}^{(i)}$ of Theorem 1 , then

$$
I\left(V_{1} ; S_{1} \mid V_{0} V_{2}\right)=\frac{1}{2} \log \frac{\left|\Sigma_{\left(V_{0}, V_{2}, S_{1}\right)}\right|\left|\Sigma_{\left(V_{0}, V_{2}, V_{1}\right)}\right|}{\left|\Sigma_{\left(V_{0}, V_{2}, V_{1}, S_{1}\right)}\right|\left|\Sigma_{\left(V_{0}, V_{2}\right)}\right|}
$$

and

$$
I\left(V_{1} ; Y \mid V_{0} V_{2}\right)=\frac{1}{2} \log \frac{\left|\Sigma_{\left(V_{0}, V_{2}, Y\right)}\right|\left|\Sigma_{\left(V_{0}, V_{2}, V_{1}\right)}\right|}{\left|\Sigma_{\left(V_{0}, V_{2}, V_{1}, Y\right)}\right|\left|\Sigma_{\left(V_{0}, V_{2}\right)}\right|} .
$$

Hence the inequality $I\left(V_{1} ; S_{1} \mid V_{0} V_{2}\right)<I\left(V_{1} ; Y \mid V_{0} V_{2}\right)$ in $\mathcal{R}^{(i)}$ is equivalent to $\frac{\left|\Sigma_{\left(V_{0}, V_{2}, S_{0}, S_{1}\right)}\right|}{\left|\Sigma_{\left(V_{0}, V_{2}, V_{1}, S_{0}, S_{1}\right)}\right|}<\frac{\left|\Sigma_{\left(V_{0}, V_{2}, Y\right)}\right|}{\left|\Sigma_{\left(V_{0}, V_{2}, V_{1}, Y\right)}\right|}$. Similarly, the last three inequalities in $\mathcal{R}^{(i)}$ are equivalent to the last three inequalities in $\mathcal{R}_{h}^{(i)}$.

\section{B. Uncoded Scheme}

Now we consider an uncoded scheme which adopts linear symbol-by-symbol encoders

$$
\begin{aligned}
& X_{1}=g_{10} S_{0}+g_{11} U_{1} \\
& X_{2}=g_{20} S_{0}+g_{22} U_{2}
\end{aligned}
$$

and MMSE (minimum mean square error) decoders (which is optimal given the encoder (31) and (32))

$$
\begin{aligned}
\hat{S}_{1} & =\mathbb{E}\left(S_{1}^{\prime} \mid Y\right) \\
& =\frac{\left(\rho_{01}\left(g_{10}+g_{20}\right)+g_{11} \sqrt{1-\rho_{01}^{2}}+g_{22} \frac{\rho_{12}-\rho_{01} \rho_{02}}{\sqrt{1-\rho_{02}^{2}}}\right) Y}{\left(g_{10}+g_{20}\right)^{2}+g_{11}^{2}+g_{22}^{2}+2 g_{11} g_{22} \frac{\rho_{12}-\rho_{01} \rho_{02}}{\sqrt{\left(1-\rho_{01}^{2}\right)\left(1-\rho_{02}^{2}\right)}}+1}, \\
\hat{S}_{2} & =\mathbb{E}\left(S_{2}^{\prime} \mid Y\right) \\
& =\frac{\left(\rho_{02}\left(g_{10}+g_{20}\right)+g_{22} \sqrt{1-\rho_{02}^{2}}+g_{11} \frac{\rho_{12}-\rho_{01} \rho_{02}}{\sqrt{1-\rho_{01}^{2}}}\right) Y}{\left(g_{10}+g_{20}\right)^{2}+g_{11}^{2}+g_{22}^{2}+2 g_{11} g_{22} \frac{\rho_{12}-\rho_{01} \rho_{02}}{\sqrt{\left(1-\rho_{01}^{2}\right)\left(1-\rho_{02}^{2}\right)}}+1}
\end{aligned}
$$

where $\left(g_{k 0}, g_{k k}\right), k=1,2$ satisfy power constraint $g_{k 0}^{2}+g_{k k}^{2} \leq$ $P_{k}, k=1,2$. Note that such uncoded scheme is a special case of hybrid coding above.

Theorem 3. The distortion pairs $\left(D_{1}^{u}, D_{2}^{u}\right)$ resulting from the described uncoded scheme are given by

$$
\begin{aligned}
& D_{1}^{u}=1-\frac{\left(\rho_{01}\left(g_{10}+g_{20}\right)+g_{11} \sqrt{1-\rho_{01}^{2}}+g_{22} \frac{\rho_{12}-\rho_{01} \rho_{02}}{\sqrt{1-\rho_{02}^{2}}}\right)^{2}}{\left(g_{10}+g_{20}\right)^{2}+g_{11}^{2}+g_{22}^{2}+2 g_{11} g_{22} \frac{\rho_{12}-\rho_{01} \rho_{02}}{\sqrt{\left(1-\rho_{01}^{2}\right)\left(1-\rho_{02}^{2}\right)}}+1}, \\
& D_{2}^{u}=1-\frac{\left(\rho_{02}\left(g_{10}+g_{20}\right)+g_{22} \sqrt{1-\rho_{02}^{2}}+g_{11} \frac{\rho_{12}-\rho_{01} \rho_{02}}{\sqrt{1-\rho_{01}^{2}}}\right)^{2}}{\left(g_{10}+g_{20}\right)^{2}+g_{11}^{2}+g_{22}^{2}+2 g_{11} g_{22} \frac{\rho_{12}-\rho_{01} \rho_{02}}{\sqrt{\left(1-\rho_{01}^{2}\right)\left(1-\rho_{02}^{2}\right)}}+1} .
\end{aligned}
$$

Hence

$\mathcal{R} \supseteq \mathcal{R}_{u}^{(i)} \triangleq\left\{\left(D_{1}, D_{2}\right):\right.$ There exist $\left(g_{k 0}, g_{k k}\right), k=1,2$ such that

$$
\left.g_{k 0}^{2}+g_{k k}^{2} \leq P_{k}, k=1,2, D_{1} \geq D_{1}^{u}, D_{2} \geq D_{2}^{u}\right\} .
$$

\section{Outer Bound}

Substitute the random variable $U$ such that (19) and (20) into the outer bound $\mathcal{R}_{2}^{(o)}$ of Theorem 1, then the following outer bound on Gaussian communication is recovered.

Theorem 4. For transmitting Gaussian source with common part over Gaussian MAC,

$\mathcal{R} \subseteq \mathcal{R}^{(o)} \triangleq\left\{\left(D_{1}, D_{2}\right):\right.$ There exist some values $0 \leq \hat{\rho} \leq 1$,

$0 \leq \hat{\rho}_{0} \leq \rho_{12 \mid 0} \triangleq \frac{\rho_{12}-\rho_{01} \rho_{02}}{\sqrt{\left(1-\rho_{01}^{2}\right)\left(1-\rho_{02}^{2}\right)}}$ such that for any $\rho_{12 \mid 0} \leq \beta_{1} \leq 1$,

$R_{S_{1} S_{2}}\left(D_{1}, D_{2}\right) \leq \frac{1}{2} \log \left(1+P_{1}+P_{2}+2 \hat{\rho} \sqrt{P_{1} P_{2}}\right)$,

$R_{S_{1} S_{2} \mid S_{0}}\left(D_{1}, D_{2}\right)$

$$
\leq \frac{1}{2} \log \left(1+\left[\frac{1-\hat{\rho}^{2}}{1-\rho_{12 \mid 0}^{2}}, 1\right]^{-}\left(P_{1}+P_{2}+2 \hat{\rho}_{0} \sqrt{P_{1} P_{2}}\right)\right),
$$

$\frac{\left(1-\rho_{01}^{2}\right)\left(1-\rho_{12 \mid 0}^{2}\right)}{D_{1}} \leq 1+\left[1-\hat{\rho}^{2}, 1-\hat{\rho}_{0}^{2}\right]^{-} P_{1}$,

$\frac{\left(1-\rho_{02}^{2}\right)\left(1-\rho_{12 \mid 0}^{2}\right)}{D_{2}} \leq 1+\left[1-\hat{\rho}^{2}, 1-\hat{\rho}_{0}^{2}\right]^{-} P_{2}$,

$\left[\frac{\left(1-\rho_{01}^{2}\right)\left(1-\beta_{1}^{2}\right)}{D_{1}}, 1\right]^{+}\left[\frac{\left(1-\rho_{02}^{2}\right)\left(1-\beta_{2}^{2}\right)}{D_{2}}, 1\right]^{+} \leq 1+$

$\left[\left(1-\theta_{1}^{2}\right) P_{1}+\left(1-\theta_{2}^{2}\right) P_{2},\left(1-\frac{\hat{\rho}_{0}^{2}}{\beta_{2}^{2}}\right) P_{1}+\left(1-\frac{\hat{\rho}_{0}^{2}}{\beta_{1}^{2}}\right) P_{2}\right]^{-}$,

$\frac{\left(1-\rho_{01}^{2}\right)\left(1-\beta_{1}^{2}\right)}{D_{1}} \leq 1+\left[1-\theta_{1}^{2}, 1-\frac{\hat{\rho}_{0}^{2}}{\beta_{2}^{2}}\right]^{-} P_{1}$,

$\frac{\left(1-\rho_{02}^{2}\right)\left(1-\beta_{2}^{2}\right)}{D_{2}} \leq 1+\left[1-\theta_{2}^{2}, 1-\frac{\hat{\rho}_{0}^{2}}{\beta_{1}^{2}}\right]^{-} P_{2}$,

for some $\theta_{1}, \theta_{2}$ such that $\left.0 \leq \theta_{1}, \theta_{2} \leq 1, \hat{\rho} \leq \theta_{1} \theta_{2}\right\}$, 
where $[x, y]^{+} \triangleq \max \{x, y\},[x, y]^{-} \triangleq \min \{x, y\}, \beta_{2}=$ $\frac{\rho_{12}-\rho_{01} \rho_{02}}{\beta_{1} \sqrt{\left(1-\rho_{01}^{2}\right)\left(1-\rho_{02}^{2}\right)}}$,

$$
\begin{aligned}
& R_{S_{1} S_{2}}\left(D_{1}, D_{2}\right) \\
& =\begin{array}{l}
p_{\hat{S}_{1} \hat{S}_{2} \mid S_{1} S_{2}}: \mathbb{E}\left(S_{k}^{\prime}-\hat{S}_{k}\right)^{2} \leq D_{k}, k=1,2 \\
=
\end{array} \\
& =\left\{\begin{array}{l}
\frac{1}{2} \log ^{+} \frac{1}{D_{1}}, \quad \text { if } \rho_{12}^{2} \geq \frac{1-D_{2}}{1-D_{1}} ; \\
\frac{1}{2} \log ^{+} \frac{1-\rho_{12}^{2}}{D_{1} D_{2}}, \quad \text { if } \rho_{12}^{2} \leq\left(1-\hat{S}_{2}\right) \\
\frac{1}{2} \log ^{+} \frac{\left.1-\rho_{12}^{2}\right)}{D_{1} D_{2}-\left(\left|\rho_{12}\right|-\sqrt{\left(1-D_{1}\right)\left(1-D_{2}\right)}\right)^{2}}, \text { otherwise }
\end{array}\right.
\end{aligned}
$$

with $\log ^{+} x \triangleq \max \{\log x, 0\}$, under the assumption that $D_{1} \leq D_{2}$, denotes the minimum sum rate needed to achieve both $D_{1}$ and $D_{2}$ at the receiver when the encoders cooperate to encode their observations [12, Thm. III.1], and

$$
\begin{aligned}
& R_{S_{1} S_{2} \mid S_{0}}\left(D_{1}, D_{2}\right) \\
& =p_{p_{\hat{S}_{1} \hat{S}_{2} \mid S_{0} S_{1} S_{2}}: \mathbb{E}\left(S_{k}^{\prime}-\hat{S}_{k}\right)^{2} \leq D_{k}, k=1,2} I\left(S_{1} S_{2} ; \hat{S}_{1} \hat{S}_{2} \mid S_{0}\right) \\
& =\inf _{p_{\hat{U}_{1} \hat{U}_{2} \mid U_{1} U_{2}}: \mathbb{E}\left(U_{k}-\hat{U}_{k}\right)^{2} \leq D_{k}, k=1,2} I\left(U_{1} U_{2} ; \hat{U}_{1} \hat{U}_{2}\right) \\
& =\left\{\begin{array}{l}
\frac{1}{2} \log ^{+} \frac{1}{D_{1}^{\prime}}, \quad \text { if } \rho_{12 \mid 0}^{2} \geq \frac{1-D_{2}^{\prime}}{1-D_{1}^{\prime}} ; \\
\frac{1}{2} \log ^{+} \frac{1-\rho_{12 \mid 0}^{2}}{D_{1}^{\prime} D_{2}^{\prime}}, \quad \text { if } \rho_{12 \mid 0}^{2} \leq\left(1-D_{1}^{\prime}\right)\left(1-D_{2}^{\prime}\right) ; \\
\frac{1}{2} \log ^{+} \frac{1-\rho_{12 \mid 0}^{2}}{D_{1}^{\prime} D_{2}^{\prime}-\left(\left|\rho_{12 \mid 0}\right|-\sqrt{\left(1-D_{1}^{\prime}\right)\left(1-D_{2}^{\prime}\right)}\right)^{2}}, \text { otherwise }
\end{array}\right.
\end{aligned}
$$

with

$$
\begin{aligned}
& D_{1}^{\prime}=\frac{D_{1}}{1-\rho_{01}^{2}}, \\
& D_{2}^{\prime}=\frac{D_{2}}{1-\rho_{02}^{2}},
\end{aligned}
$$

under the assumption that $D_{1}^{\prime} \leq D_{2}^{\prime}$, denotes the minimum sum rate needed to achieve both $D_{1}$ and $D_{2}$ at the receiver when the side information $S_{0}$ is available at both the encoders and the decoder and the encoders cooperate to encode their observations with help of $S_{0}$.

The proof of Theorem 4 is given in Appendix B. The Maximal Correlation Theory (Hirschfeld-Gebelein-Rényi maximal correlation) is exploited in the proof. When $\rho_{01}=\rho_{02}=0$, Theorem 4 can recover the outer bound without common part [12, Thm. IV.1]. Besides, Theorem 4 can be extended to any other source-channel pair by following similar steps to the proof.

When specialized to the symmetric case, Theorem 4 reduces to the following result.
Corollary 1. In the symmetric case,

$\mathcal{R}_{\text {sym }} \subseteq \mathcal{R}_{\text {sym }}^{(o)} \triangleq\{D:$ There exist some values $0 \leq \hat{\rho} \leq 1$,

$0 \leq \hat{\rho}_{0} \leq \rho_{12 \mid 0} \triangleq \frac{\rho_{12}-\rho_{01} \rho_{02}}{\sqrt{\left(1-\rho_{01}^{2}\right)\left(1-\rho_{02}^{2}\right)}}$ such that for any $\rho_{12 \mid 0} \leq \beta_{1} \leq 1$,

$R_{S_{1} S_{2}}(D, D) \leq \frac{1}{2} \log (1+2(1+\hat{\rho}) P)$,

$R_{S_{1} S_{2} \mid S_{0}}(D, D) \leq \frac{1}{2} \log \left(1+\left[\frac{1-\hat{\rho}^{2}}{1-\rho_{12 \mid 0}^{2}}, 1\right]^{-} \cdot 2\left(1+\hat{\rho}_{0}\right) P\right)$,

$\frac{\left(1-\rho_{01}^{2}\right)\left(1-\rho_{12 \mid 0}^{2}\right)}{D} \leq 1+\left[1-\hat{\rho}^{2}, 1-\hat{\rho}_{0}^{2}\right]^{-} P$,

$\frac{\left(1-\rho_{02}^{2}\right)\left(1-\rho_{12 \mid 0}^{2}\right)}{D} \leq 1+\left[1-\hat{\rho}^{2}, 1-\hat{\rho}_{0}^{2}\right]^{-} P$,

$\left[\frac{\left(1-\rho_{01}^{2}\right)\left(1-\beta_{1}^{2}\right)}{D}, 1\right]^{+}\left[\frac{\left(1-\rho_{02}^{2}\right)\left(1-\beta_{2}^{2}\right)}{D}, 1\right]^{+}$

$\leq 1+\left[2-\theta_{1}^{2}-\theta_{2}^{2}, 2-\frac{\hat{\rho}_{0}^{2}}{\beta_{2}^{2}}-\frac{\hat{\rho}_{0}^{2}}{\beta_{1}^{2}}\right]^{-} P$,

$\frac{\left(1-\rho_{01}^{2}\right)\left(1-\beta_{1}^{2}\right)}{D} \leq 1+\left[1-\theta_{1}^{2}, 1-\frac{\hat{\rho}_{0}^{2}}{\beta_{2}^{2}}\right]^{-} P$,

$\frac{\left(1-\rho_{02}^{2}\right)\left(1-\beta_{2}^{2}\right)}{D} \leq 1+\left[1-\theta_{2}^{2}, 1-\frac{\hat{\rho}_{0}^{2}}{\beta_{1}^{2}}\right]^{-} P$,

for some $\theta_{1}, \theta_{2}$ such that $\left.0 \leq \theta_{1}, \theta_{2} \leq 1, \hat{\rho} \leq \theta_{1} \theta_{2}\right\}$,

where

$$
R_{S_{1} S_{2}}(D, D)=\left\{\begin{array}{l}
\frac{1}{2} \log ^{+} \frac{1-\rho_{12}^{2}}{D^{2}}, \text { if }\left|\rho_{12}\right| \leq 1-D \\
\frac{1}{2} \log ^{+} \frac{1+\left|\rho_{12}\right|}{2 D-\left(1-\left|\rho_{12}\right|\right)}, \text { otherwise }
\end{array}\right.
$$

and

$$
R_{S_{1} S_{2} \mid S_{0}}(D, D)=\left\{\begin{array}{l}
\frac{1}{2} \log ^{+} \frac{1-\rho_{12 \mid 0}^{2}}{D^{\prime 2}}, \text { if }\left|\rho_{12 \mid 0}\right| \leq 1-D^{\prime} \\
\frac{1}{2} \log ^{+} \frac{1+\left|\rho_{12 \mid 0}\right|}{2 D^{\prime}-\left(1-\left|\rho_{12 \mid 0}\right|\right)}, \text { otherwise }
\end{array}\right.
$$

with

$$
D^{\prime}=\frac{D}{1-\rho_{01}^{2}} .
$$

Fig. 3 illustrates the various bounds on the achievable distortion.

\section{CONCLUding REMARKS}

In this paper, we focused on the joint source-channel coding problem of sending memoryless correlated sources with common part over memoryless multiple access channel, and developed an inner bound and two outer bounds for this problem. The inner bound is achieved by a unified hybrid coding scheme with common part, and as special cases, it can recover the performance of existing hybrid coding without common part. Similarly, our outer bound can also recover several outer bounds in the literature. When specialized to transmitting Gaussian sources over Gaussian MAC, the inner bound and outer bound are used to generate a new inner bound and a new outer bound, which can recover the best known inner bound and outer bound without common part in the literature.

It is worth noting that in our results, two kinds of common informations are involved. They are respectively in sense of Gács-Körner-Witsenhausen common information [13], [14], 


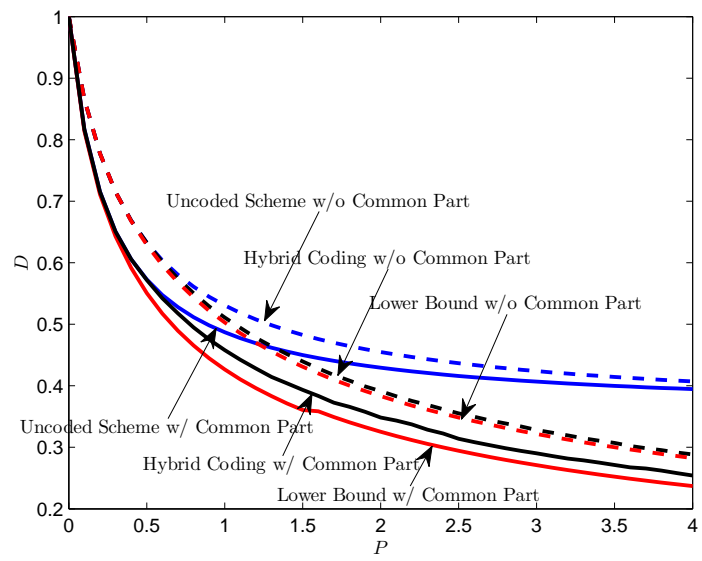

Fig. 3. Distortion bounds in the symmetric case for sending Gaussian sources over Gaussian MAC. Uncoded Scheme w/ Common Part, Hybrid Coding w/ Common Part, and Lower Bound w/ Common Part are the bounds for the case with common part where $\rho_{12}=0.3, \rho_{01}=\rho_{02}=0.8$. They respectively correspond to the inner bound in Theorem 3 , the inner bound in Theorem 2, the outer bound in Corollary 1. Uncoded Scheme w/o Common Part, Hybrid Coding w/o Common Part, and Lower Bound w/o Common Part are the bounds for the case with no common part where $\rho_{12}=0.3, \rho_{01}=$ $\rho_{02}=0$.

and in sense of Wyner's common information [15]. In our problem, the Gács-Körner-Witsenhausen common information, i.e., the common part, has been exploited to improve the performance of communication system, and the Wyner's common information has been exploited to obtain the outer bounds. Besides, correlation ratio and maximal correlation coefficient are also utilized to derive the outer bound for Gaussian communication case. These concepts and tools are expected to be exploited to derive achievability and converse results for other problems in network information theory.

\section{APPENDIX A PROOF OF THEOREM 1}

\section{A. Inner Bound}

We use the hybrid coding shown in Fig. 2 to prove the inner bound.

Codebook Generation: Fix conditional pmf $p_{V_{0} \mid S_{0}} p_{V_{1} \mid S_{1}, V_{0}} p_{V_{2} \mid S_{2}, V_{0}}$, encoding functions $x_{k}\left(v_{0}, v_{k}, s_{k}\right), k=1,2$ and decoding functions $\hat{s}_{k}\left(v_{0}, v_{1}, v_{2}, y\right), k=1,2$ that satisfy all the inequalities in the inner bound (5). Randomly and independently generate a set of sequences $v_{0}^{n}\left(m_{0}\right), m_{0} \in\left[1: 2^{n r_{0}}\right]$, with each distributed according to $\prod_{i=1}^{n} p_{V_{0}}\left(v_{0, i}\right)$. For $k=1,2$ and for each $m_{0} \in\left[1: 2^{n r_{0}}\right]$, randomly and independently generate a set of sequences $v_{k}^{n}\left(m_{0}, m_{k}\right), m_{k} \in\left[1: 2^{n r_{k}}\right]$, with each distributed according to $\prod_{i=1}^{n} p_{V_{k} \mid V_{0}}\left(v_{k, i} \mid v_{0, i}\left(m_{0}\right)\right)$. The codebook

$$
\begin{aligned}
\mathcal{C}=\{ & \left(v_{0}^{n}\left(m_{0}\right), v_{1}^{n}\left(m_{0}, m_{1}\right), v_{2}^{n}\left(m_{0}, m_{2}\right)\right): \\
& \left.\left(m_{0}, m_{1}, m_{2}\right) \in\left[1: 2^{n r_{0}}\right] \times\left[1: 2^{n r_{1}}\right] \times\left[1: 2^{n r_{2}}\right]\right\} .
\end{aligned}
$$

is revealed to both the encoders and the decoder.
Encoding: We use joint typicality encoding. Let $\epsilon>\epsilon_{0}$. Given $s_{0}^{n}$, both encoders 1 and 2 find the smallest index $m_{0}$ such that $\left(s_{0}^{n}, v_{0}^{n}\left(m_{0}\right)\right) \in \mathcal{T}_{\epsilon_{0}}^{(n)}$. If there is no such index, let $m_{0}=1$. For $k=1,2$, given $s_{k}^{n}$ and $v_{0}^{n}\left(m_{0}\right)$, encoder $k$ finds the smallest index $m_{k}$ such that $\left(s_{0}^{n}, s_{k}^{n}, v_{0}^{n}\left(m_{0}\right), v_{k}^{n}\left(m_{0}, m_{k}\right)\right) \in \mathcal{T}_{\epsilon}^{(n)}$. If there is no such index, let $m_{k}=1$. Then the encoder $k$ transmits the signal

$$
x_{k, i}=x_{k}\left(v_{0, i}\left(m_{0}\right), v_{k, i}\left(m_{0}, m_{k}\right), s_{k, i}\right), 1 \leq i \leq n .
$$

Decoding: We use joint typicality decoding. Let $\epsilon^{\prime}>\epsilon$. Upon receiving signal $y^{n}$, the decoder of the receiver finds the smallest index vector $\left(\hat{m}_{0}, \hat{m}_{1}, \hat{m}_{2}\right)$ such that

$$
\left(v_{0}^{n}\left(\hat{m}_{0}\right), v_{1}^{n}\left(\hat{m}_{0}, \hat{m}_{1}\right), v_{2}^{n}\left(\hat{m}_{0}, \hat{m}_{2}\right), y^{n}\right) \in \mathcal{T}_{\epsilon^{\prime}}^{(n)}
$$

If there is no such index vector, let $\left(\hat{m}_{0}, \hat{m}_{1}, \hat{m}_{2}\right)=(1,1,1)$. The decoder reconstructs the sources as for $k=1,2$,

$\hat{s}_{k, i}=\hat{s}_{k}\left(v_{0, i}\left(\hat{m}_{0}\right), v_{1, i}\left(\hat{m}_{0}, \hat{m}_{1}\right), v_{2, i}\left(\hat{m}_{0}, \hat{m}_{2}\right), y_{i}\right), 1 \leq i \leq n$.

Analysis of Expected Distortion: We bound the distortion averaged over $\left(S_{1}^{n}, S_{2}^{n}\right)$, and the random choice of the codebook $\mathcal{C}$. Define the "error" event

$$
\begin{aligned}
& \mathcal{E}= \\
& \left\{\left(S_{0}^{n}, S_{1}^{n}, S_{2}^{n}, V_{0}^{n}\left(\hat{M}_{0}\right), V_{1}^{n}\left(\hat{M}_{0}, \hat{M}_{1}\right), V_{2}^{n}\left(\hat{M}_{0}, \hat{M}_{2}\right), Y^{n}\right) \notin \mathcal{T}_{\epsilon^{\prime}}^{(n)}\right\} .
\end{aligned}
$$

Then we have

$$
\mathcal{E} \subseteq \mathcal{E}_{0} \cup \mathcal{E}_{1} \cup \mathcal{E}_{2} \cup \mathcal{E}_{3} \cup \mathcal{E}_{4} \cup \mathcal{E}_{5}
$$

where

$$
\begin{aligned}
& \mathcal{E}_{0}=\left\{\left(S_{0}^{n}, V_{0}^{n}\left(m_{0}\right)\right) \notin \mathcal{T}_{\epsilon_{0}}^{(n)} \text { for all } m_{0}\right\}, \\
& \mathcal{E}_{1}=\left\{\left(S_{0}^{n}, S_{1}^{n}, V_{0}^{n}\left(M_{0}\right), V_{1}^{n}\left(M_{0}, m_{1}\right)\right) \notin \mathcal{T}_{\epsilon}^{(n)} \text { for all } m_{1}\right\}, \\
& \mathcal{E}_{2}=\left\{\left(S_{0}^{n}, S_{2}^{n}, V_{0}^{n}\left(M_{0}\right), V_{2}^{n}\left(M_{0}, m_{2}\right)\right) \notin \mathcal{T}_{\epsilon}^{(n)} \text { for all } m_{2}\right\}, \\
& \mathcal{E}_{3}=\left\{\left(S_{0}^{n}, S_{1}^{n}, S_{2}^{n}, V_{0}^{n}\left(M_{0}\right), V_{1}^{n}\left(M_{0}, M_{1}\right), V_{2}^{n}\left(M_{0}, M_{2}\right)\right) \notin \mathcal{T}_{\epsilon_{1}}^{(n)}\right\}, \\
& \mathcal{E}_{4}=\left\{\left(S_{0}^{n}, S_{1}^{n}, S_{2}^{n}, V_{0}^{n}\left(M_{0}\right), V_{1}^{n}\left(M_{0}, M_{1}\right), V_{2}^{n}\left(M_{0}, M_{2}\right), Y^{n}\right) \notin \mathcal{T}_{\epsilon^{\prime}}^{(n)}\right\}, \\
& \mathcal{E}_{5}=\left\{\left(V_{0}^{n}\left(m_{0}^{\prime}\right), V_{1}^{n}\left(m_{0}^{\prime}, m_{1}^{\prime}\right), V_{2}^{n}\left(m_{0}^{\prime}, m_{2}^{\prime}\right), Y^{n}\right) \in \mathcal{T}_{\epsilon^{\prime}}^{(n)}\right. \\
& \text { for some } \left.\left(m_{0}^{\prime}, m_{1}^{\prime}, m_{2}^{\prime}\right) \neq\left(M_{0}, M_{1}, M_{2}\right)\right\}
\end{aligned}
$$

for some $\epsilon_{1}$ such that $\epsilon_{0}<\epsilon<\epsilon_{1}<\epsilon^{\prime}$. Using union bound, we have

$$
\begin{aligned}
\mathbb{P}(\mathcal{E}) & \leq \mathbb{P}\left(\mathcal{E}_{0}\right)+\mathbb{P}\left(\mathcal{E}_{0}^{c} \cap \mathcal{E}_{1}\right)+\mathbb{P}\left(\mathcal{E}_{0}^{c} \cap \mathcal{E}_{2}\right) \\
& +\mathbb{P}\left(\mathcal{E}_{0}^{c} \cap \mathcal{E}_{1}^{c} \cap \mathcal{E}_{2}^{c} \cap \mathcal{E}_{3}\right)+\mathbb{P}\left(\mathcal{E}_{3}^{c} \cap \mathcal{E}_{4}\right)+\mathbb{P}\left(\mathcal{E}_{5}\right)
\end{aligned}
$$

Now we claim that if all the inequalities in the inner bound (5) hold, then $\mathbb{P}(\mathcal{E})$ tends to zero as $n \rightarrow \infty$. Before proving it, we show that this claim implies the distortions in the inner bound (5) are achievable. The expected distortions are bounded 
by

$$
\begin{aligned}
& \limsup _{n \rightarrow \infty} \mathbb{E} d_{k}\left(S_{k}^{n}, \hat{S}_{k}^{n}\right) \\
& =\limsup _{n \rightarrow \infty}\left(\mathbb{P}\left(\mathcal{E}_{4, k}\right) \mathbb{E}\left[d_{k}\left(S_{k}^{n}, \hat{S}_{k}^{n}\right) \mid \mathcal{E}_{4, k}\right]\right. \\
& \left.+\mathbb{P}\left(\mathcal{E}_{4, k}^{c}\right) \mathbb{E}\left[d_{k}\left(S_{k}^{n}, \hat{S}_{k}^{n}\right) \mid \mathcal{E}_{4, k}^{c}\right]\right) \\
& =\limsup _{n \rightarrow \infty} \mathbb{E}\left[d_{k}\left(S_{k}^{n}, \hat{S}_{k}^{n}\right) \mid \mathcal{E}_{4, k}^{c}\right] \\
& \leq\left(1+\epsilon^{\prime}\right) \mathbb{E} d_{k}\left(S_{k}, \hat{S}_{k}\right) \\
& \leq\left(1+\epsilon^{\prime}\right) D_{k},
\end{aligned}
$$

for $k=1,2$, where (42) follows from typical average lemma [9]. Therefore, the desired distortions are achieved for sufficiently small $\epsilon^{\prime}$.

Next we turn back to prove the claim above. Following from covering lemma [9, Sec. 3.7], the first three terms of (39), $\mathbb{P}\left(\mathcal{E}_{0}\right)+\mathbb{P}\left(\mathcal{E}_{0}^{c} \cap \mathcal{E}_{1}\right)+\mathbb{P}\left(\mathcal{E}_{0}^{c} \cap \mathcal{E}_{2}\right)$, vanishes as $n \rightarrow \infty$ if

$$
r_{0}>I\left(V_{0} ; S_{0}\right)
$$

and according to Markov lemma [9, Sec. 12.1.1], the fourth item tends to zero as $n \rightarrow \infty$ if

$$
r_{k}>I\left(V_{k} ; S_{k} \mid V_{0}\right), k=1,2 .
$$

Then by conditional typicality lemma [9, Sec. 2.5], the fifth item tends to zero as $n \rightarrow \infty$.

Now we focus on the last term of (39). $\mathcal{E}_{5}$ can be writen as

$$
\mathcal{E}_{5}=\mathcal{E}_{51} \cup \mathcal{E}_{52} \cup \mathcal{E}_{53} \cup \mathcal{E}_{54},
$$

where

$$
\begin{gathered}
\mathcal{E}_{51}=\left\{\left(V_{0}^{n}\left(m_{0}^{\prime}\right), V_{1}^{n}\left(m_{0}^{\prime}, m_{1}^{\prime}\right), V_{2}^{n}\left(m_{0}^{\prime}, m_{2}^{\prime}\right), Y^{n}\right) \in \mathcal{T}_{\epsilon^{\prime}}^{(n)}\right. \\
\text { for some } \left.m_{0}^{\prime} \neq M_{0}, m_{1}^{\prime} \neq M_{1}, m_{2}^{\prime} \neq M_{2}\right\}, \\
\mathcal{E}_{52}=\left\{\left(V_{0}^{n}\left(M_{0}\right), V_{1}^{n}\left(M_{0}, m_{1}^{\prime}\right), V_{2}^{n}\left(M_{0}, m_{2}^{\prime}\right), Y^{n}\right) \in \mathcal{T}_{\epsilon^{\prime}}^{(n)}\right. \\
\text { for some } \left.m_{1}^{\prime} \neq M_{1}, m_{2}^{\prime} \neq M_{2}\right\}, \\
\mathcal{E}_{53}=\left\{\left(V_{0}^{n}\left(M_{0}\right), V_{1}^{n}\left(M_{0}, M_{1}\right), V_{2}^{n}\left(M_{0}, m_{2}^{\prime}\right), Y^{n}\right) \in \mathcal{T}_{\epsilon^{\prime}}^{(n)}\right. \\
\left.\quad \text { for some } m_{2}^{\prime} \neq M_{2}\right\}, \\
\mathcal{E}_{54}=\left\{\left(V_{0}^{n}\left(M_{0}\right), V_{1}^{n}\left(M_{0}, m_{1}^{\prime}\right), V_{2}^{n}\left(M_{0}, M_{2}\right), Y^{n}\right) \in \mathcal{T}_{\epsilon^{\prime}}^{(n)}\right. \\
\text { for some } \left.m_{1}^{\prime} \neq M_{1}\right\},
\end{gathered}
$$

Using union bound we have

$$
\mathbb{P}\left(\mathcal{E}_{5}\right) \leq \mathbb{P}\left(\mathcal{E}_{51}\right)+\mathbb{P}\left(\mathcal{E}_{52}\right)+\mathbb{P}\left(\mathcal{E}_{53}\right)+\mathbb{P}\left(\mathcal{E}_{54}\right) .
$$

Following similar steps to the proof of [7, Thm. 1], one can prove $\mathbb{P}\left(\mathcal{E}_{51}\right)$ vanishes as $n \rightarrow \infty$ if

$$
r_{0}+r_{1}+r_{2}<I\left(V_{0} V_{1} V_{2} ; Y\right)+I\left(V_{1} ; V_{2} \mid V_{0}\right),
$$

$\mathbb{P}\left(\mathcal{E}_{52}\right)$ vanishes as $n \rightarrow \infty$ if

$$
r_{1}+r_{2}<I\left(V_{1} V_{2} ; Y \mid V_{0}\right)+I\left(V_{1} ; V_{2} \mid V_{0}\right),
$$

$\mathbb{P}\left(\mathcal{E}_{53}\right)$ vanishes as $n \rightarrow \infty$ if

$$
r_{2}<I\left(V_{2} ; Y \mid V_{0} V_{1}\right)
$$

and $\mathbb{P}\left(\mathcal{E}_{54}\right)$ vanishes as $n \rightarrow \infty$ if

$$
r_{1}<I\left(V_{1} ; Y \mid V_{0} V_{2}\right) \text {. }
$$

Combining (44), (45), and (48)-(51) leads to the sufficient condition, which completes the proof of the inner bound.

\section{B. Outer Bound}

For fixed $p_{U_{[1: L]} \mid S_{1}, S_{2}}$, we introduce a set of auxiliary random variables $U_{[1: L]}^{n}$ that follow $\prod_{i=1}^{n} p_{U_{[1: L]} \mid S_{1}, S_{2}}\left(u_{[1: L], i} \mid s_{1, i}, s_{2, i}\right)$. Then the Markov chain $U_{[1: L]}^{n} \rightarrow\left(S_{1}^{n}, S_{2}^{n}\right) \rightarrow\left(X_{1}^{n}, X_{2}^{n}\right) \rightarrow Y^{n} \rightarrow\left(\hat{S}_{1}^{n}, \hat{S}_{2}^{n}\right)$ holds. Assume $\mathcal{A} \subseteq[1: L]$. Next, we derive a lower bound for $I\left(S_{1}^{n} S_{2}^{n} ; Y^{n} \mid U_{\mathcal{A}}^{\bar{n}}\right)$.

$$
\begin{aligned}
& I\left(S_{1}^{n} S_{2}^{n} ; Y^{n} \mid U_{\mathcal{A}}^{n}\right) \\
&= \sum_{t=1}^{n} I\left(S_{1, t} S_{2, t} ; Y^{n} \mid U_{\mathcal{A}}^{n} S_{1}^{t-1} S_{2}^{t-1}\right) \\
&= \sum_{t=1}^{n} H\left(S_{1, t} S_{2, t} \mid U_{\mathcal{A}}^{n} S_{1}^{t-1} S_{2}^{t-1}\right) \\
& \quad \quad H\left(S_{1, t} S_{2, t} \mid Y^{n} U_{\mathcal{A}}^{n} S_{1}^{t-1} S_{2}^{t-1}\right) \\
&=\sum_{t=1}^{n} H\left(S_{1, t} S_{2, t} \mid U_{\mathcal{A}, t}\right)-H\left(S_{1, t} S_{2, t} \mid Y^{n} U_{\mathcal{A}}^{n} S_{1}^{t-1} S_{2}^{t-1}\right) \\
&=\sum_{t=1}^{n} I\left(S_{1, t} S_{2, t} ; Y^{n} U_{\mathcal{A}}^{n} S_{1}^{t-1} S_{2}^{t-1} \mid U_{\mathcal{A}, t}\right) \\
& \geq \sum_{t=1}^{n} I\left(S_{1, t} S_{2, t} ; \hat{S}_{1, t} \hat{S}_{2, t} \mid U_{\mathcal{A}, t}\right) \\
&=n I\left(S_{1, Q} S_{2, Q} ; \hat{S}_{1, Q} \hat{S}_{2, Q} \mid U_{\mathcal{A}, Q} Q\right) \\
&=n I\left(S_{1, Q} S_{2, Q} ; \hat{S}_{1, Q} \hat{S}_{2, Q} Q \mid U_{\mathcal{A}, Q}\right) \\
& \geq n I\left(S_{1, Q} S_{2, Q} ; \hat{S}_{1, Q} \hat{S}_{2, Q} \mid U_{\mathcal{A}, Q}\right) \\
&=n I\left(S_{1} S_{2} ; \hat{S}_{1} \hat{S}_{2} \mid U_{\mathcal{A}}\right),
\end{aligned}
$$

where $Q$ is a time-sharing random variable uniformly distributed $[1: n]$ and independent of all other random variables, and in (60), $S_{k} \triangleq S_{k, Q}, \hat{S}_{k} \triangleq \hat{S}_{k, Q}, U_{l} \triangleq U_{l, Q}, k=1,2,1 \leq$ $l \leq L$.

Now, we turn to upper-bounding $I\left(S_{1}^{n} S_{2}^{n} ; Y^{n} \mid U_{\mathcal{A}}^{n}\right)$.

$$
\begin{aligned}
I & \left(S_{1}^{n} S_{2}^{n} ; Y^{n} \mid U_{\mathcal{A}}^{n}\right) \\
\leq & I\left(X_{1}^{n} X_{2}^{n} ; Y^{n} \mid U_{\mathcal{A}}^{n}\right) \\
= & \sum_{t=1}^{n} I\left(Y_{t} ; X_{1}^{n} X_{2}^{n} \mid U_{\mathcal{A}}^{n} Y^{t-1}\right) \\
\leq & \sum_{t=1}^{n} I\left(Y_{t} ; X_{1}^{n} X_{2}^{n} Y^{t-1} \mid U_{\mathcal{A}}^{n}\right) \\
= & \sum_{t=1}^{n} I\left(Y_{t} ; X_{1, t} X_{2, t} \mid U_{\mathcal{A}}^{n}\right) \\
= & n I\left(Y_{Q} ; X_{1, Q} X_{2, Q} \mid U_{\mathcal{A}}^{n} Q\right), \\
= & n I\left(Y ; X_{1} X_{2} \mid U_{\mathcal{A}}^{n} Q\right),
\end{aligned}
$$

where (64) follows from $\left(X_{1}^{n}, X_{2}^{n}, Y^{t-1}\right) \rightarrow\left(X_{1, t}, X_{2, t}\right) \rightarrow$ $Y_{t}, Q$ is the time-sharing random variable defined above, and $Y \triangleq Y_{Q}, X_{k} \triangleq X_{k, Q}, k=1,2$.

Combine (60) and (66), then we have

$I\left(S_{1} S_{2} ; \hat{S}_{1} \hat{S}_{2} \mid U_{\mathcal{A}}\right) \leq I\left(X_{1} X_{2} ; Y \mid U_{\mathcal{A}}^{n} Q\right)$ for any $\mathcal{A} \subseteq[1: L]$. 
In addition, $\left(Q, S_{1}^{n}, S_{2}^{n}, X_{1}, X_{2}, Y\right)$ follows the distribution $p_{Q} \prod p_{S_{1}, S_{2}}\left(s_{1, i}, s_{2, i}\right) p_{U_{[1: L]} \mid S_{1}, S_{2}}\left(u_{[1: L], i} \mid s_{1, i}, s_{2, i}\right)$ $p_{X_{1} \mid S_{1}^{n}, Q} p_{X_{2} \mid S_{2}^{n}, Q} p_{Y \mid X_{1}, X_{2}}$. This completes the proof of the outer bound $\mathcal{R}_{1}^{(o)}$.

\section{APPENDIX B \\ PROOF OF THEOREM 4}

Before proving Theorem 4, we need introduce several correlations and their properties, including correlation coefficient, correlation ratio, maximal correlation coefficient, as well as the corresponding conditional correlations.

Definition 5. For any random variables $W_{1}$ and $W_{2}$ with alphabets $\mathcal{W}_{1} \subseteq \mathbb{R}$ and $\mathcal{W}_{2} \subseteq \mathbb{R}$, the (Pearson) correlation coefficient of $W_{1}$ and $W_{2}$ is defined by

$$
\rho\left(W_{1}, W_{2}\right)=\frac{\operatorname{cov}\left(W_{1}, W_{2}\right)}{\sqrt{\operatorname{var}\left(W_{1}\right)} \sqrt{\operatorname{var}\left(W_{2}\right)}} .
$$

Similarly, the conditional correlation coefficient of $W_{1}$ and $W_{2}$ given another random variable $W_{0}$ is defined by

$$
\rho\left(W_{1}, W_{2} \mid W_{0}\right)=\frac{\mathbb{E}\left[\operatorname{cov}\left(W_{1}, W_{2} \mid W_{0}\right)\right]}{\sqrt{\mathbb{E}\left[\operatorname{var}\left(W_{1} \mid W_{0}\right)\right]} \sqrt{\mathbb{E}\left[\operatorname{var}\left(W_{2} \mid W_{0}\right)\right]}} .
$$

Definition 6. For any random variables $W_{1}$ and $W_{2}$ with alphabets $\mathcal{W}_{1} \subseteq \mathbb{R}$ and $\mathcal{W}_{2}$, the correlation ratio of $W_{1}$ on $W_{2}$ is defined by

$$
\theta\left(W_{1}, W_{2}\right)=\sup _{f} \rho\left(W_{1}, f\left(W_{2}\right)\right)
$$

where the supremum is taken over all the functions $f: \mathcal{W}_{2} \mapsto$ $\mathbb{R}$ satisfying

$$
0<\mathbb{E}\left[f^{2}\left(W_{2}\right)\right]<\infty
$$

Similarly, the conditional correlation ratio of $W_{1}$ on $W_{2}$ given another random variable $W_{0}$ is defined by

$$
\theta\left(W_{1}, W_{2} \mid W_{0}\right)=\sup _{f} \rho\left(W_{1}, f\left(W_{2}, W_{0}\right) \mid W_{0}\right),
$$

where the supremum is taken over all the functions $f: \mathcal{W}_{2} \times$ $\mathcal{W}_{0} \mapsto \mathbb{R}$ satisfying

$$
0<\mathbb{E}\left[f^{2}\left(W_{2}, W_{0}\right)\right]<\infty .
$$

Definition 7. For any random variables $W_{1}$ and $W_{2}$ with alphabets $\mathcal{W}_{1}$ and $\mathcal{W}_{2}$, the maximal correlation coefficient of $W_{1}$ and $W_{2}$ is defined by

$$
\rho_{m}\left(W_{1}, W_{2}\right)=\sup _{f_{1}, f_{2}} \rho\left(f_{1}\left(W_{1}\right), f_{2}\left(W_{2}\right)\right),
$$

where the supremum is taken over all the functions $f_{k}: \mathcal{W}_{k} \mapsto$ $\mathbb{R}$ for $k=1,2$, satisfying

$$
0<\mathbb{E}\left[f_{k}^{2}\left(W_{k}\right)\right]<\infty
$$

Moreover, the conditional maximal correlation coefficient of $W_{1}$ and $W_{2}$ given another random variable $W_{0}$ is defined by

$$
\rho_{m}\left(W_{1}, W_{2} \mid W_{0}\right)=\sup _{f_{1}, f_{2}} \rho\left(f_{1}\left(W_{1}, W_{0}\right), f_{2}\left(W_{2}, W_{0}\right) \mid W_{0}\right),
$$

where the supremum is taken over all the functions $f_{k}: \mathcal{W}_{k} \times$ $\mathcal{W}_{0} \mapsto \mathbb{R}$ for $k=1,2$, satisfying

$$
0<\mathbb{E}\left[f_{k}^{2}\left(W_{k}, W_{0}\right)\right]<\infty .
$$

Lemma 1. [19] For any random variables $W_{0}, W_{1}$ and $W_{2}$, (conditional) correlation coefficient, (conditional) correlation ratio, and (conditional) maximal correlation coefficient have the following properties:

$$
0 \leq\left|\rho\left(W_{1}, W_{2}\right)\right| \leq \theta\left(W_{1}, W_{2}\right) \leq \rho_{m}\left(W_{1}, W_{2}\right) \leq 1 ;
$$

$$
\begin{gathered}
0 \leq\left|\rho\left(W_{1}, W_{2} \mid W_{0}\right)\right| \leq \theta\left(W_{1}, W_{2} \mid W_{0}\right) \\
\quad \leq \rho_{m}\left(W_{1}, W_{2} \mid W_{0}\right) \leq 1 ; \\
\theta\left(W_{1}, W_{2} W_{0}\right) \geq \theta\left(W_{1}, W_{0}\right) ; \\
\rho_{m}\left(W_{1}, W_{2} W_{0}\right) \geq \rho_{m}\left(W_{1}, W_{0}\right) ; \\
\theta\left(W_{1}, W_{2}\right)=\sqrt{\frac{\operatorname{var}\left(\mathbb{E}\left[W_{1} \mid W_{2}\right]\right)}{\operatorname{var}\left(W_{1}\right)}} \\
=\sqrt{1-\frac{\mathbb{E}\left[\operatorname{var}\left(W_{1} \mid W_{2}\right)\right]}{\operatorname{var}\left(W_{1}\right)}} ;
\end{gathered}
$$

$$
\begin{aligned}
\theta\left(W_{1}, W_{2} \mid W_{0}\right) & =\sqrt{\frac{\mathbb{E}\left[\operatorname{var}\left(\mathbb{E}\left[W_{1} \mid W_{2} W_{0}\right] \mid W_{0}\right)\right]}{\mathbb{E}\left[\operatorname{var}\left(W_{1} \mid W_{0}\right)\right]}} \\
& =\sqrt{1-\frac{\mathbb{E}\left[\operatorname{var}\left(W_{1} \mid W_{2} W_{0}\right)\right]}{\mathbb{E}\left[\operatorname{var}\left(W_{1} \mid W_{0}\right)\right]}} ;
\end{aligned}
$$

$$
\begin{aligned}
\rho_{m}\left(W_{1}, W_{2}\right) & =\sup _{f} \sqrt{\frac{\operatorname{var}\left(\mathbb{E}\left[f\left(W_{1}\right) \mid W_{2}\right]\right)}{\operatorname{var}\left(f\left(W_{1}\right)\right)}} \\
& =\sup _{f} \sqrt{1-\frac{\mathbb{E}\left[\operatorname{var}\left(f\left(W_{1}\right) \mid W_{2}\right)\right]}{\operatorname{var}\left(f\left(W_{1}\right)\right)}} ;
\end{aligned}
$$

$$
\begin{aligned}
\rho_{m}\left(W_{1}, W_{2} \mid W_{0}\right) & =\sup _{f} \sqrt{\frac{\mathbb{E}\left[\operatorname{var}\left(\mathbb{E}\left[f\left(W_{1}, W_{0}\right) \mid W_{2} W_{0}\right] \mid W_{0}\right)\right]}{\mathbb{E}\left[\operatorname{var}\left(f\left(W_{1}, W_{0}\right) \mid W_{0}\right)\right]}} \\
& =\sup _{f} \sqrt{1-\frac{\mathbb{E}\left[\operatorname{var}\left(f\left(W_{1}, W_{0}\right) \mid W_{2} W_{0}\right)\right]}{\mathbb{E}\left[\operatorname{var}\left(f\left(W_{1}, W_{0}\right) \mid W_{0}\right)\right]}} ;
\end{aligned}
$$

$$
\begin{aligned}
1-\theta^{2} & \left(W_{1}, W_{2} W_{0}\right) \\
= & \left(1-\theta^{2}\left(W_{1}, W_{0}\right)\right)\left(1-\theta^{2}\left(W_{1}, W_{2} \mid W_{0}\right)\right) ;
\end{aligned}
$$

and

$$
\begin{aligned}
& 1-\theta^{2}\left(W_{1}, W_{2} W_{0} \mid Z\right) \\
& \quad=\left(1-\theta^{2}\left(W_{1}, W_{0} \mid Z\right)\right)\left(1-\theta^{2}\left(W_{1}, W_{2} \mid W_{0} Z\right)\right) .
\end{aligned}
$$

Besides, some other remarkable properties are also needed in proving Theorem 4.

Lemma 2. [14, Thm. 1] For a sequence of pairs of independent random variables $\left(W_{1, i}, W_{2, i}\right)_{i=1}^{n}$, we have

$$
\rho_{m}\left(W_{1}^{n}, W_{2}^{n}\right) \leq \sup _{1 \leq i \leq n} \rho_{m}\left(W_{1, i}, W_{2, i}\right)
$$

where $W_{k}^{n}=\left(W_{k, 1}, W_{k, 2}, \cdots, W_{k, n}\right)$ for $k=1,2$.

Lemma 3. [16, Sec. IV, Lem. 10.2] For jointly Gaussian random variables $W_{0}, W_{1}$ and $W_{2}$, we have

$$
\begin{aligned}
\rho_{m}\left(W_{1}, W_{2}\right) & =\left|\rho\left(W_{1}, W_{2}\right)\right|, \\
\rho_{m}\left(W_{1}, W_{2} \mid W_{0}\right) & =\left|\rho\left(W_{1}, W_{2} \mid W_{0}\right)\right| .
\end{aligned}
$$


Lemma 4 (Data Processing Inequality). [19] If random variable $W$ and non-degenerate random variables $X, Y, Z$ form a Markov chain $X \rightarrow(Y, W) \rightarrow Z$, then

$$
\begin{aligned}
\rho(X, Z \mid W) & \leq \theta(X, Y \mid W) \theta(Z, Y \mid W) \\
\theta(X, Z \mid W) & \leq \theta(X, Y \mid W) \rho_{m}(Z, Y \mid W) \\
\rho_{m}(X, Z \mid W) & \leq \rho_{m}(X, Y \mid W) \rho_{m}(Z, Y \mid W) .
\end{aligned}
$$

Moreover, the equalities hold in (85)-(87), if $(X, Y, W)$ and $(Z, Y, W)$ have the same distribution. In particular, if $W$ is degenerate, then

$$
\begin{aligned}
\rho(X, Z) & \leq \theta(X, Y) \theta(Z, Y), \\
\theta(X, Z) & \leq \theta(X, Y) \rho_{m}(Z, Y), \\
\rho_{m}(X, Z) & \leq \rho_{m}(X, Y) \rho_{m}(Z, Y) .
\end{aligned}
$$

Now we use $\mathcal{R}_{2}^{(o)}$ to prove Theorem 4 . For $\mathcal{R}_{2}^{(o)}$, denote $\hat{\rho}$ as the correlation coefficient between $X_{1}$ and $X_{2}$, i.e., $\hat{\rho} \triangleq$ $\rho\left(X_{1}, X_{2}\right)$, and $\theta_{k}$ as correlation ratio of $X_{k}$ on $\left(S_{0}^{n}, U^{n}, Q\right)$ , i.e.,

$$
\theta_{k} \triangleq \theta\left(X_{k}, S_{0}^{n} U^{n} Q\right), k=1,2 .
$$

It should hold that $0 \leq \hat{\rho}, \theta_{1}, \theta_{2} \leq 1$. Observe that in $\mathcal{R}_{2}^{(o)}$, $X_{1} \rightarrow\left(S_{1}^{n}, Q\right) \rightarrow\left(S_{0}^{n}, U^{n}, Q\right) \rightarrow\left(S_{2}^{n}, Q\right) \rightarrow X_{2}$ holds. Hence from Lemma 4 , we have

$$
\hat{\rho} \leq \theta_{1} \theta_{2}
$$

From Property (76) of Lemma 1, we have

$$
\mathbb{E}\left[\operatorname{var}\left(X_{k} \mid S_{0}^{n} U^{n} Q\right)\right]=\left(1-\theta_{k}^{2}\right) \mathbb{E}\left[\operatorname{var}\left(X_{k}\right)\right], k=1,2 . \quad \text { (93) }
$$

In addition, denote $\hat{\rho}_{0}=\rho\left(X_{1}, X_{2} \mid S_{0}^{n} Q\right)$, $\rho_{12 \mid 0}=\rho\left(S_{1}, S_{2} \mid S_{0}\right)=\frac{\rho_{12}-\rho_{01} \rho_{02}}{\sqrt{\left(1-\rho_{01}^{2}\right)\left(1-\rho_{02}^{2}\right)}}$ and $\theta_{k}^{\prime}=\theta\left(X_{k}, U^{n} \mid S_{0}^{n} Q\right), k=1,2$. Then utilizing Lemmas 2,3 and 4 , we have

$$
\begin{aligned}
\hat{\rho}_{0} \leq \rho_{m} & \left(S_{1}^{n}, S_{2}^{n} \mid S_{0}^{n} Q\right)=\rho_{m}\left(S_{1}, S_{2} \mid S_{0}\right)=\rho_{12 \mid 0}, \\
\hat{\rho}_{0} & \leq \theta\left(X_{1}, S_{2}^{n} \mid S_{0}^{n} Q\right) \\
& \leq \theta\left(X_{1}, U^{n} \mid S_{0}^{n} Q\right) \rho_{m}\left(S_{2}^{n}, U^{n} \mid S_{0}^{n} Q\right) \\
& =\theta_{1}^{\prime} \beta_{2},
\end{aligned}
$$

and

$$
\hat{\rho}_{0} \leq \theta_{2}^{\prime} \beta_{1}
$$

Now based on the inequalities above and utilizing the outer bound $\mathcal{R}_{2}^{(o)}$ of Theorem 1, we can obtain a sequence of desired results. Specifically, Combining the inequality $I\left(S_{1} S_{2} ; \hat{S}_{1} \hat{S}_{2}\right) \leq I\left(X_{1} X_{2} ; Y \mid Q\right)$ in $\mathcal{R}_{2}^{(o)}$ with

$$
I\left(S_{1} S_{2} ; \hat{S}_{1} \hat{S}_{2}\right) \geq R_{S_{1} S_{2}}\left(D_{1}, D_{2}\right)
$$

and

$$
\begin{aligned}
I\left(X_{1} X_{2} ; Y \mid Q\right)= & h(Y \mid Q)-h\left(Y \mid X_{1} X_{2}\right) \\
\leq & h(Y)-h\left(Y \mid X_{1} X_{2}\right) \\
\leq & \frac{1}{2} \log \left(1+\operatorname{var}\left(X_{1}+X_{2}\right)\right) \\
= & \frac{1}{2} \log \left(1+\operatorname{var}\left(X_{1}\right)+\operatorname{var}\left(X_{2}\right)\right. \\
& \left.\quad+2 \rho\left(X_{1}, X_{2}\right) \sqrt{\operatorname{var}\left(X_{1}\right) \operatorname{var}\left(X_{2}\right)}\right) \\
\leq & \frac{1}{2} \log \left(1+P_{1}+P_{2}+2 \hat{\rho} \sqrt{P_{1} P_{2}}\right)
\end{aligned}
$$

gives

$$
R_{S_{1} S_{2}}\left(D_{1}, D_{2}\right) \leq \frac{1}{2} \log \left(1+P_{1}+P_{2}+2 \hat{\rho} \sqrt{P_{1} P_{2}}\right) .
$$

In addition, from Property (80) of Lemma 1, we have

$$
\begin{aligned}
1-\theta^{2}\left(X_{1}, S_{0}^{n} Q\right) & =\frac{1-\theta^{2}\left(X_{1}, X_{2} S_{0}^{n} Q\right)}{1-\theta^{2}\left(X_{1}, X_{2} \mid S_{0}^{n} Q\right)} \\
& \leq \min \left(\frac{1-\hat{\rho}^{2}}{1-\rho_{12 \mid 0}^{2}}, 1\right),
\end{aligned}
$$

where the inequality (107) follows from

$$
\theta\left(X_{1}, X_{2} S_{0}^{n} Q\right) \geq \theta\left(X_{1}, X_{2}\right) \geq \rho\left(X_{1}, X_{2}\right),
$$

and

$$
\theta\left(X_{1}, X_{2} \mid S_{0}^{n} Q\right) \leq \rho_{m}\left(X_{1}, X_{2} \mid S_{0}^{n} Q\right) \leq \rho_{12 \mid 0} .
$$

Then combining the inequality $I\left(S_{1} S_{2} ; \hat{S}_{1} \hat{S}_{2} \mid S_{0}\right) \leq$ $I\left(X_{1} X_{2} ; Y \mid S_{0}^{n} Q\right)$ in $\mathcal{R}_{2}^{(o)}$ with

$$
I\left(S_{1} S_{2} ; \hat{S}_{1} \hat{S}_{2} \mid S_{0}\right) \geq R_{S_{1} S_{2} \mid S_{0}}\left(D_{1}, D_{2}\right)
$$

and

$$
\begin{aligned}
& I\left(X_{1} X_{2} ; Y \mid S_{0}^{n} Q\right) \\
& =h\left(Y \mid S_{0}^{n} Q\right)-h\left(Y \mid X_{1} X_{2}\right) \\
& \leq \frac{1}{2} \log \left(1+\mathbb{E} \operatorname{var}\left(X_{1}+X_{2} \mid S_{0}^{n} Q\right)\right) \\
& =\frac{1}{2} \log \left(1+\mathbb{E} \operatorname{var}\left(X_{1} \mid S_{0}^{n} Q\right)+\mathbb{E} \operatorname{var}\left(X_{2} \mid S_{0}^{n} Q\right)\right. \\
& \left.\quad+2 \rho\left(X_{1}, X_{2} \mid S_{0}^{n} Q\right) \sqrt{\mathbb{E} v a r\left(X_{1} \mid S_{0}^{n} Q\right) \mathbb{E} \operatorname{var}\left(X_{2} \mid S_{0}^{n} Q\right)}\right) \\
& \leq \frac{1}{2} \log \left(1+\left(1-\theta^{2}\left(X_{1}, S_{0}^{n} Q\right)\right) P_{1}+\left(1-\theta^{2}\left(X_{2}, S_{0}^{n} Q\right)\right) P_{2}\right. \\
& \left.\quad+2 \hat{\rho}_{0} \sqrt{\left(1-\theta^{2}\left(X_{1}, S_{0}^{n} Q\right)\right)\left(1-\theta^{2}\left(X_{2}, S_{0}^{n} Q\right)\right) P_{1} P_{2}}\right) \\
& \leq \frac{1}{2} \log \left(1+\min \left(\frac{1-\hat{\rho}^{2}}{1-\rho_{12 \mid 0}^{2}}, 1\right)\left(P_{1}+P_{2}+2 \hat{\rho}_{0} \sqrt{P_{1} P_{2}}\right)\right)
\end{aligned}
$$

gives

$$
\begin{aligned}
& R_{S_{1} S_{2} \mid S_{0}}\left(D_{1}, D_{2}\right) \\
& \leq \frac{1}{2} \log \left(1+\min \left(\frac{1-\hat{\rho}^{2}}{1-\rho_{12 \mid 0}^{2}}, 1\right)\left(P_{1}+P_{2}+2 \hat{\rho}_{0} \sqrt{P_{1} P_{2}}\right)\right) .
\end{aligned}
$$

Similarly, the last five inequalities in (33) can be obtained as well. This completes the proof. 


\section{REFERENCES}

[1] L. Ozarow, "On a source-coding problem with two channels and three receivers," Bell Syst. Tech. Journal, vol. 59, pp. 1909-1921, Dec. 1980.

[2] A. B. Wagner, S. Tavildar, and P. Viswanath, "Rate region of the quadratic Gaussian two-encoder source-coding problem," IEEE Trans. Inf. Theory, vol. 54, no. 5, pp. 1938-1961, May 2008.

[3] Z. Reznic, M. Feder, and R. Zamir, "Distortion bounds for broadcasting with bandwidth expansion," IEEE Trans. Inf. Theory, vol. 52, no. 8, pp. 3778-3788, Aug. 2006

[4] L. Yu, H. Li, and W. Li, "Distortion bounds for source broadcast over degraded channel," in Proc. IEEE Int. Symp. Inf. Theory (ISIT), Barcelona, Spain, Jul. 2016, pp. 1834-1838.

[5] L. Yu, H. Li, and W. Li, "Distortion bounds for source broadcast problem," Submitted to IEEE Trans. Inf. Theory, 2016.

[6] K. Khezeli, and J. Chen "Outer bounds on the admissible source region for broadcast channels with correlated sources," IEEE Trans. Inf. Theory, vol. 61, pp. 4616-4629, Sep. 2015.

[7] P. Minero, S. H. Lim, and Y. H. Kim, "A unified approach to hybrid coding," IEEE Trans. Inf. Theory, vol. 61, no. 4, pp. 1509-1523, 2015.

[8] A. B. Wagner, B. G. Kelly, and Y. Altuğ, "Distributed rate-distortion with common components," IEEE Trans. Inf. Theory, vol. 57, no. 7, pp. 4035-4057, Aug. 2011.

[9] A. El Gamal and Y.-H. Kim, Network Information Theory. Cambridge University Press, 2011., vol. 60, pp. 6760-6786, Nov. 2014.

[10] T. M. Cover, A. El Gamal, and M. Salehi, "Multiple access channels with arbitrarily correlated sources," IEEE Trans. Inf. Theory, vol. 26, no. 6, pp. 648-657, Nov. 1980.

[11] D. Slepian and J. K. Wolf, "A coding theorem for multiple access channels with correlated sources," Bell Syst. Tech. J., vol. 52, no. 7, pp. 1037-1076, Sep. 1973.

[12] A. Lapidoth and S. Tinguely, "Sending a bivariate Gaussian over a Gaussian MAC," IEEE Trans. Inf. Theory, vol. 56, no. 6, pp. 2714-2752, 2010.

[13] P. Gács and J. Körner, "Common information is far less than mutual information," Probl. Contr. Inform. Theory, vol. 2, no. 2, pp. 149-162, 1973.

[14] H. S. Witsenhausen, "On sequences of pairs of dependent random variables," SIAM J. Appl. Math., vol. 28, no. 1, pp. 100-113, Jan. 1975.

[15] A. Wyner, "The common information of two dependent random variables," IEEE Trans. Inf. Theory, vol. 21, no. 2, pp. 163-179, Mar. 1975.

[16] Y. A. Rozanov, Stationary Random Processes. San Francisco, CA: Holden-Day, 1967.

[17] W. Kang and S. Ulukus, "A new data processing inequality and its applications in distributed source and channel coding," IEEE Trans. on Inf. Theory, vol. 57, no. 1, pp. 56-69, Jan. 2011.

[18] A. Lapidoth and M. Wigger, "A necessary condition for the transmissibility of correlated sources over a MAC," in Proc. IEEE Int. Symp. Inf. Theory (ISIT), Barcelona, Spain, Jul. 2016, pp. 2024-2028.

[19] L. Yu, H. Li, and C. W. Chen "Generalized common information: Common information extraction and private sources synthesis," [Online]. Available: https://arxiv.org/abs/1610.09289. 